\title{
A re-assessment of the transition-metal free Suzuki-type coupling methodology
}

\author{
Riina K. Arvela ${ }^{a}$, Nicholas. E. Leadbeater ${ }^{a, *}$, Michael S. Sangi $^{\mathrm{a}}$ and Victoria. J. Williams ${ }^{a}$, \\ Patricia Granados, ${ }^{\mathrm{b}}$ Robert Singer ${ }^{\mathrm{b}}$ \\ a) Department of Chemistry, University of Connecticut, Unit 3060, 55 North Eagleville Road, Storrs, \\ CT 06269-3060,USA. E-mail: nicholas.leadbeater@uconn.edu \\ b) Department of Chemistry, Saint Mary's University, Halifax, Nova Scotia B3H 3C3, Canada
}

\section{Supporting Information}

${ }^{1} \mathrm{H},{ }^{13} \mathrm{C}$ NMR and MS data for the compounds prepared S1-S46 


\section{Spectroscopic data}

Compound 1: 4,4'- Diacetylbiphenyl (Table 1, entry 1): ${ }^{1} \mathrm{H}$ NMR $\left(\mathrm{CDCl}_{3}\right) \delta 8.05(\mathrm{~d}, \mathrm{~J}=$ $8.3 \mathrm{~Hz}, 4 \mathrm{H}), 7.71(\mathrm{~d}, \mathrm{~J}=8.3 \mathrm{~Hz}, 4 \mathrm{H}), 2.64(\mathrm{~s}, 6 \mathrm{H}) ;{ }^{13} \mathrm{C} \mathrm{NMR}\left(\mathrm{CDCl}_{3}\right)$ d 197.6, 144.3, 136.6, $129.0,127.4,26.7$.

Compound 2: 4- Acetyl- 4'- methoxybiphenyl (Table 1, entries 2 and 3): ${ }^{1} \mathrm{H}$ NMR $\left(\mathrm{CDCl}_{3}\right) \delta 8.01$ (d, J= $\left.8.2 \mathrm{~Hz}, 2 \mathrm{H}\right), 7.65(\mathrm{~d}, \mathrm{~J}=8.2 \mathrm{~Hz}, 2 \mathrm{H}), 7.58(\mathrm{~d}, \mathrm{~J}=8.6 \mathrm{~Hz}, 2 \mathrm{H}), 7.00(\mathrm{~d}, \mathrm{~J}=$ $8.6 \mathrm{~Hz}, 2 \mathrm{H}), 3.87(\mathrm{~s}, 3 \mathrm{H}), 2.63$ (s, 3H); ${ }^{13} \mathrm{C}$ NMR $\left(\mathrm{CDCl}_{3}\right)$ d 197.7, 159.9, 145.4, 135.3, 132.3, $129.0,128.4,126.6,114.4,55.4,26.6$.

Compound 3: 4- Methoxy- 4'- methylbiphenyl (Table 1, entry 4): ${ }^{1} \mathrm{H}$ NMR $\left(\mathrm{CDCl}_{3}\right) \delta$ $7.59(\mathrm{~d}, \mathrm{~J}=8.8 \mathrm{~Hz}, 2 \mathrm{H}), 7.53(\mathrm{~d}, \mathrm{~J}=8.1 \mathrm{~Hz}, 2 \mathrm{H}), 7.30(\mathrm{~d}, \mathrm{~J}=7.9 \mathrm{~Hz}, 2 \mathrm{H}), 7.04(\mathrm{~d}, \mathrm{~J}=8.8 \mathrm{~Hz}$, 2H), 3.90 (s, 3H), 2.46 (s, 3H); ${ }^{13} \mathrm{C}$ NMR $\left(\mathrm{CDCl}_{3}\right)$ ठ 159.0, 138.1, 136.4, 133.8, 129.5, 128.0, 126.7, 114.3, 55.4, 21.1.

Compound 4: 4- Acetyl- 2'- methylbiphenyl (Table 1, entry 6): ${ }^{1} \mathrm{H}$ NMR ( $\left.\mathrm{CDCl}_{3}\right) \delta 8.02$ $(\mathrm{d}, \mathrm{J}=8.3 \mathrm{~Hz}, 2 \mathrm{H}), 7.43(\mathrm{~d}, \mathrm{~J}=8.3 \mathrm{~Hz}, 2 \mathrm{H}), 7.22-7.31(\mathrm{~m}, 4 \mathrm{H}), 2.65(\mathrm{~s}, 3 \mathrm{H}), 2.28(\mathrm{~s}, 3 \mathrm{H}) ;{ }^{13} \mathrm{C}$ NMR $\left(\mathrm{CDCl}_{3}\right) \delta 197.8,147.0,140.8,135.6,135.2,130.5,129.5,129.5,128.2,127.9,125.9$, $26.7,20.4$.

Compound 5: N-(4'-acetyl-biphenyl-4-yl)-acetamide (Table 1, entry 7): ${ }^{1} \mathrm{H}$ NMR (DMSO) ठ 10.05 (s, 1H), 7.97 (d, J= 8.3 Hz, 2H), 7.76 (d, J= 8.4 Hz, 2H), 7.67 (s, 4H), 2.56 (s, 3H), $2.04(\mathrm{~s}, 3 \mathrm{H}) ;{ }^{13} \mathrm{C}$ NMR $\left(\mathrm{CDCl}_{3}\right)$ o 197.9, 168.9, 144.5, 140.1, 135.6, 133.6, 129.4, 127.7, 126.7, 119.8, 27.2, 24.5; HRMS (FAB) calcd for $\mathrm{C}_{16} \mathrm{H}_{16} \mathrm{O}_{2} \mathrm{~N}(\mathrm{M}+1) 254.3085$, found 254.1181. 
Compound 6: N-(4'-methoxy-biphenyl-4-yl)-acetamide (Table 1, entry 8): ${ }^{1} \mathrm{H}$ NMR

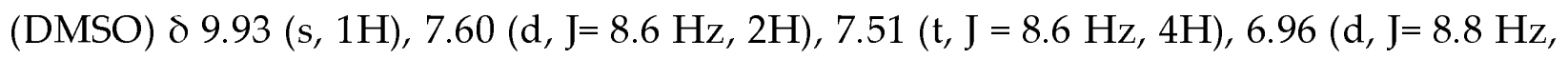
2H), 3.75 (s, 3H), $2.02(\mathrm{~s}, 3 \mathrm{H}) ;{ }^{13} \mathrm{C}$ NMR $\left(\mathrm{CDCl}_{3}\right)$ o 168.7, 159.0, 138.6, 134.9, 132.7, 127.7, $126.8,119.8,114.8,55.6,24.5$.

Compound 7: 4- Acetyl- trans- stilbene (Table 2, entry 1 and Table 5, entry 7): $1 \mathrm{H}$

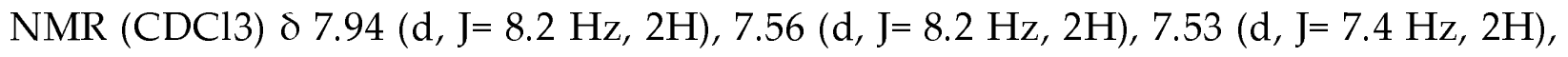
7.36-7.40 (m, 2H), 7.26-7.32 (m, 1H), 7.21 (d, J=16.3 Hz, 1H), 7.11 (d, J=16.3 Hz, 1H), 2.59 (s, 3H); ${ }^{13} \mathrm{C}$ NMR $\left(\mathrm{CDCl}_{3}\right) \delta$ 197.4, 142.0, 136.7, 136.0, 131.5, 128.9, 128.8, 128.3, 127.4, $126.9,126.5,26.6$.

Compound 8: 4- Methoxy- trans -stilbene (Table 2, entry 2 and Table 5, entries 2, 6 and 8): $1 \mathrm{H}$ NMR $(\mathrm{CDCl} 3) \delta$ 7.46-7.51 $(\mathrm{m}, 4 \mathrm{H}), 7.36(\mathrm{t}, \mathrm{J}=7.6 \mathrm{~Hz}, 2 \mathrm{H}), 7.23-7.27(\mathrm{~m}, 1 \mathrm{H})$, $7.08(\mathrm{~d}, \mathrm{~J}=16.3 \mathrm{~Hz}, 1 \mathrm{H}), 6.99$ (d, J= $16.3 \mathrm{~Hz}, 1 \mathrm{H}), 6.92(\mathrm{~d}, \mathrm{~J}=8.7 \mathrm{~Hz}, 2 \mathrm{H}), 3.84(\mathrm{~s}, 3 \mathrm{H}) ;{ }^{13} \mathrm{C}$ NMR $\left(\mathrm{CDCl}_{3}\right)$ o 159.3, 137.7, 130.2, 128.7, 128.2, 127.7, 127.2, 126.7, 126.3, 114.2, 55.3.

Compound 9: 2- Methyl- trans -stilbene (Table 2, entry 3): 1H NMR (CDCl3) $\delta 7.61$ (d, $\mathrm{J}=7.0 \mathrm{~Hz}, 1 \mathrm{H}), 7.54(\mathrm{~d}, \mathrm{~J}=7.4 \mathrm{~Hz}, 2 \mathrm{H}), 7.20-7.40(\mathrm{~m}, 7 \mathrm{H}), 7.02(\mathrm{~d}, \mathrm{~J}=16.2 \mathrm{~Hz}, 1 \mathrm{H}), 2.45(\mathrm{~s}$, $3 \mathrm{H}) ;{ }^{13} \mathrm{C} \mathrm{NMR}\left(\mathrm{CDCl}_{3}\right) \delta$ 137.7, 136.4, 135.8, 130.4, 130.0, 128.7, 127.6, 127.6, 126.6, 126.2, 125.4, 19.9.

Compound 10: 2- Cyano- trans -stilbene (Table 2, entry 4): 1H NMR (CDCl3) $\delta 7.80(\mathrm{~d}$, $\mathrm{J}=8.1 \mathrm{~Hz}, 1 \mathrm{~Hz}), 7.64-7.70(\mathrm{~m}, 1 \mathrm{H}), 7.56-7.59(\mathrm{~m}, 3 \mathrm{H}), 7.30-7.48(\mathrm{~m}, 6 \mathrm{H}) ;{ }^{13} \mathrm{C}$ NMR $\left(\mathrm{CDCl}_{3}\right)$ o 140.6, 136.2, 133.4, 133.1, 132.8, 128.9, 128.8, 127.5, 127.1, 125.3, 124.1, 111.3 
Compound 11: 4- Fluoro- trans -stilbene (Table 2, entry 5): 1H NMR (CDCl3) $\delta$ 7.47$7.52(\mathrm{~m}, 4 \mathrm{H}), 7.37(\mathrm{t}, \mathrm{J}=7.6 \mathrm{~Hz}, 2 \mathrm{H}), 7.25-7.32(\mathrm{~m}, 1 \mathrm{H}), 7.00-7.10(\mathrm{~m}, 4 \mathrm{H}) ;{ }^{13} \mathrm{C}$ NMR $\left(\mathrm{CDCl}_{3}\right) \delta 162.4$ (d), 137.2, 133.5 (d), 128.7, 128.5, 128.0 (d), 127.7, 127.5, 126.5, 115.6 (d).

Compound 12: 4- Amino- trans -stilbene (Table 2, entry 6 and Table 5, entry 5): $1 \mathrm{H}$ $\operatorname{NMR}(\mathrm{CDCl} 3) \delta 7.48(\mathrm{~d}, \mathrm{~J}=7.3 \mathrm{~Hz}, 2 \mathrm{H}), 7.32-7.36(\mathrm{~m}, 2 \mathrm{H}), 7.22(\mathrm{tt}, \mathrm{J}=7.3 \mathrm{~Hz}, \mathrm{~J}=1.0 \mathrm{~Hz}$ 1H), $7.03(\mathrm{~d}, \mathrm{~J}=16.3 \mathrm{~Hz}, 1 \mathrm{H}), 6.93(\mathrm{~d}, \mathrm{~J}=16.3 \mathrm{~Hz}, 1 \mathrm{H}), 6.68(\mathrm{~d}, \mathrm{~J}=8.5 \mathrm{~Hz}, 2 \mathrm{H}), 3.68$ (bs, 2H); ${ }^{13} \mathrm{C}$ NMR $\left(\mathrm{CDCl}_{3}\right) \delta$ d 146.2, 138.0, 128.7, 128.6, 128.4, 128.1, 127.8, 126.9, 126.1, 125.1, 115.2 .

Compound 13: (E)-1-[4-Hex-1-enyl)phenyl]ethanone (Scheme 2): 1H NMR (CDCl3) $\delta$ $7.87(\mathrm{~d}, \mathrm{~J}=8.3 \mathrm{~Hz}, 2 \mathrm{H}), 7.39$ (d, J= $8.3 \mathrm{~Hz}, 2 \mathrm{H}), 6.34-6.43(\mathrm{~m}, 2 \mathrm{H}), 2.57(\mathrm{~s}, 3 \mathrm{H}), 2.21-2.24$ $(\mathrm{m}, 2 \mathrm{H}), 1.42-1.49(\mathrm{~m}, 2 \mathrm{H}), 1.34-1.40(\mathrm{~m}, 2 \mathrm{H}), 0.93(\mathrm{t}, \mathrm{J}=7.2 \mathrm{~Hz}, 3 \mathrm{H}) ;{ }^{13} \mathrm{C} \mathrm{NMR}\left(\mathrm{CDCl}_{3}\right) \delta$ $197.5,142.7,135.4,134.5,128.9,128.7,125.9,32.8,31.3,26.5,22.3,13.9$.

Compound 14: Trans -stilbene (Scheme 2 and Table 5, entry 3): 1H NMR (CDCl3) $\delta$ $7.54(\mathrm{~d}, \mathrm{~J}=7.6 \mathrm{~Hz}, 4 \mathrm{H}), 7.38(\mathrm{t}, \mathrm{J}=7.6 \mathrm{~Hz}, 4 \mathrm{H}), 7.28(\mathrm{t}, \mathrm{J}=7.3 \mathrm{~Hz}, 2 \mathrm{H}), 7.14(\mathrm{~s}, 2 \mathrm{H}) ;{ }^{13} \mathrm{C}$ NMR $\left(\mathrm{CDCl}_{3}\right) \delta$ 137.4, 128.7, 127.6, 126.5.

Compound 15: (E)-1,4-Diphenyl-buta-1,3-diene (Scheme 2): 1H NMR (CDCl3) ठ 7.48 $(\mathrm{d}, \mathrm{J}=7.5 \mathrm{~Hz}, 4 \mathrm{H}), 7.37$ (t, J= 7.5 Hz, 4H), 7.27 (t, J = 7.1 Hz, 2H), $6.99(\mathrm{~d}, \mathrm{~J}=14.4 \mathrm{~Hz}, 2 \mathrm{H})$, $6.71(\mathrm{~d}, \mathrm{~J}=14.2 \mathrm{~Hz}, 2 \mathrm{H}) ;{ }^{13} \mathrm{C}$ NMR $\left(\mathrm{CDCl}_{3}\right) \delta$ 137.4, 132.9, 129.3, 128.7, 127.6, 126.4 .

Compound 16: 4- Acetylbiphenyl (Tables 3 and 4): 1H NMR (CDCl3) $\delta 8.04$ (d, J = 8.4 $\mathrm{Hz}, 2 \mathrm{H}), 7.69$ (d, J = 8.4 Hz, 2H), $7.63(\mathrm{~d}, \mathrm{~J}=7.5 \mathrm{~Hz}, 2 \mathrm{H}), 7.47(\mathrm{~d}, \mathrm{~J}=7.5 \mathrm{~Hz}, 2 \mathrm{H}), 7.41(\mathrm{~d}, \mathrm{~J}$ $=7.4 \mathrm{~Hz}, 1 \mathrm{H}), 2.64(\mathrm{~s}, 3 \mathrm{H}) ;{ }^{13} \mathrm{C} \mathrm{NMR}\left(\mathrm{CDCl}_{3}\right) \delta$ 197.7, 145.8, 139.9, 135.9, 129.0, 128.9, $128.2,127.3,127.2,26.7$. 
Compound 17: 4- Methylbiphenyl (Tables 3 and 4): 1H NMR (CDCl3) $\delta 7.64(\mathrm{~d}, \mathrm{~J}=7.1$ $\mathrm{Hz}, 2 \mathrm{H}), 7.55(\mathrm{~d}, \mathrm{~J}=8.1 \mathrm{~Hz}, 2 \mathrm{H}), 7.48(\mathrm{t}, \mathrm{J}=7.5 \mathrm{~Hz}, 2 \mathrm{H}), 7.37(\mathrm{t}, \mathrm{J}=7.3 \mathrm{~Hz}, 1 \mathrm{H}), 7.30(\mathrm{~d}, \mathrm{~J}=$ $7.9 \mathrm{~Hz}, 2 \mathrm{H}), 2.45(\mathrm{~s}, 3 \mathrm{H})) ;{ }^{13} \mathrm{C} \mathrm{NMR}\left(\mathrm{CDCl}_{3}\right) \delta$ 141.2, 138.4, 137.0, 129.5, 128.7, 127.0, 127.0, 21.1.

Compound 18: 4- Methoxybiphenyl (Tables 3 and 4): $1 \mathrm{H}$ NMR (CDCl3) $\delta 7.53-7.58$ $(\mathrm{m}, 4 \mathrm{H}), 7.43(\mathrm{t}, \mathrm{J}=7.7 \mathrm{~Hz}, 2 \mathrm{H}), 7.32(\mathrm{t}, \mathrm{J}=7.4 \mathrm{~Hz}, 1 \mathrm{H}), 6.99(\mathrm{~d}, \mathrm{~J}=8.8 \mathrm{~Hz}, 2 \mathrm{H}), 3.86(\mathrm{~s}$, $3 \mathrm{H}) ;{ }^{13} \mathrm{C} \mathrm{NMR}\left(\mathrm{CDCl}_{3}\right) \delta$ 159.2, 140.9, 133.8, 128.7, 128.2, 126.8, 126.7, 114.2, 55.4.

Compound 19: 4- Methyl- trans -stilbene (Table 5, entries 1 and 9): 1H NMR (CDCl3) $\delta 7.53(\mathrm{~d}, \mathrm{~J}=7.7 \mathrm{~Hz}, 2 \mathrm{H}), 7.44(\mathrm{~d}, \mathrm{~J}=8.0 \mathrm{~Hz}, 2 \mathrm{H}), 7.38(\mathrm{t}, \mathrm{J}=7.6 \mathrm{~Hz}, 2 \mathrm{H}), 7.27(\mathrm{t}, \mathrm{J}=7.3 \mathrm{~Hz}$, $1 \mathrm{H}), 7.19(\mathrm{~d}, \mathrm{~J}=8.0 \mathrm{~Hz}, 2 \mathrm{H}), 7.10(\mathrm{~s}, 2 \mathrm{H}), 2.39(\mathrm{~s}, 3 \mathrm{H}) ;{ }^{13} \mathrm{C} \mathrm{NMR}\left(\mathrm{CDCl}_{3}\right) \delta$ 137.6, 137.5, 134.6, 129.4, 128.7, 127.7, 127.4, 126.5, 126.4, 21.3.

Compound 20: 4- Chloro- trans -stilbene (Table 5, entry 4): 1H NMR (CDCl3) $\delta 7.52$ (d, $\mathrm{J}=7.6 \mathrm{~Hz}, 2 \mathrm{H}), 7.45(\mathrm{~d}, \mathrm{~J}=8.3 \mathrm{~Hz}, 2 \mathrm{H}), 7.27-7.40(\mathrm{~m}, 5 \mathrm{H}), 7.08(\mathrm{~s}, 2 \mathrm{H}) ;{ }^{13} \mathrm{C} \mathrm{NMR}\left(\mathrm{CDCl}_{3}\right) \delta$ $137.0,135.9,133.2,129.4,128.9,128.8,127.9,127.7,127.4,126.6$. 

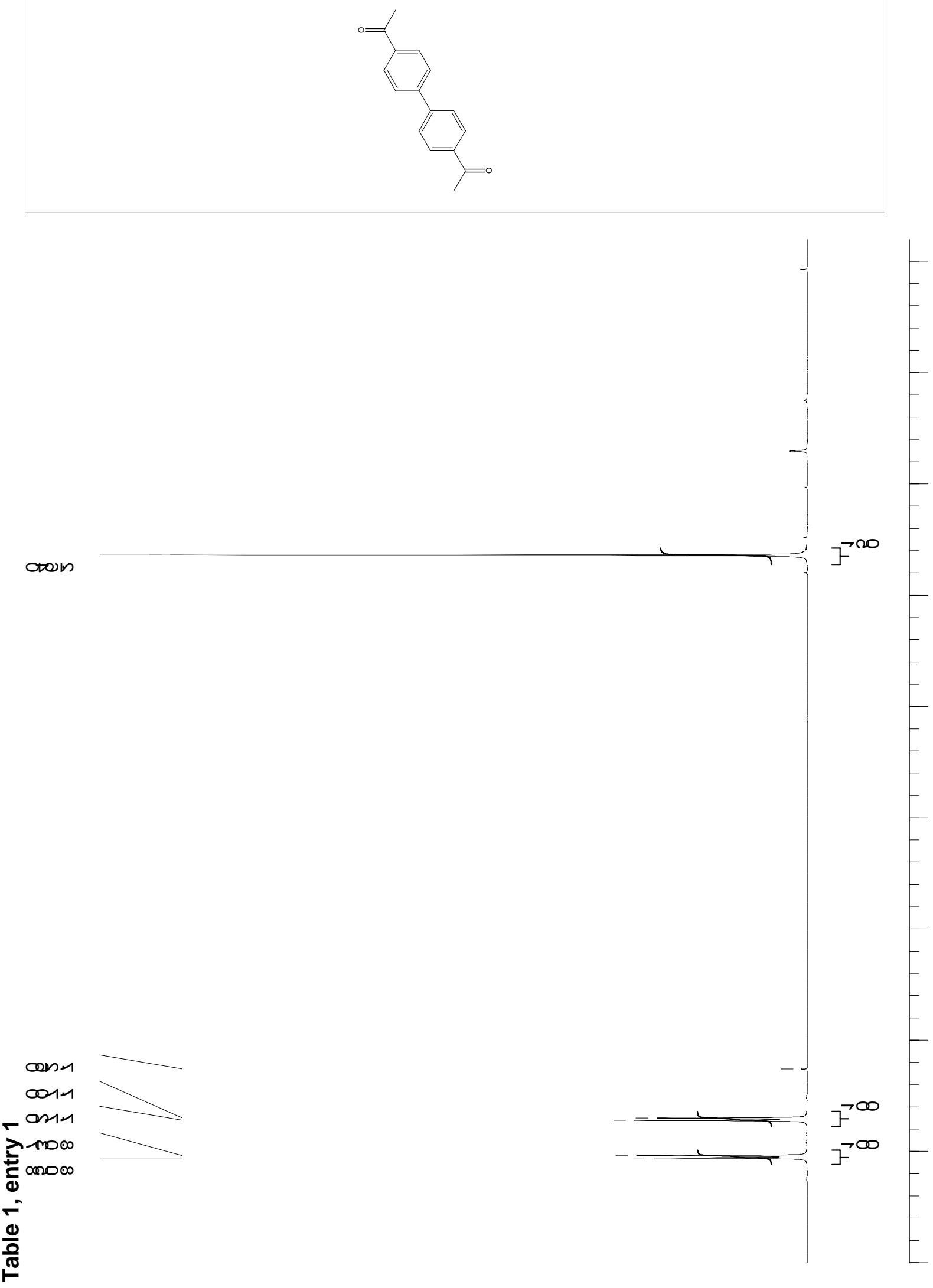


$$
1
$$



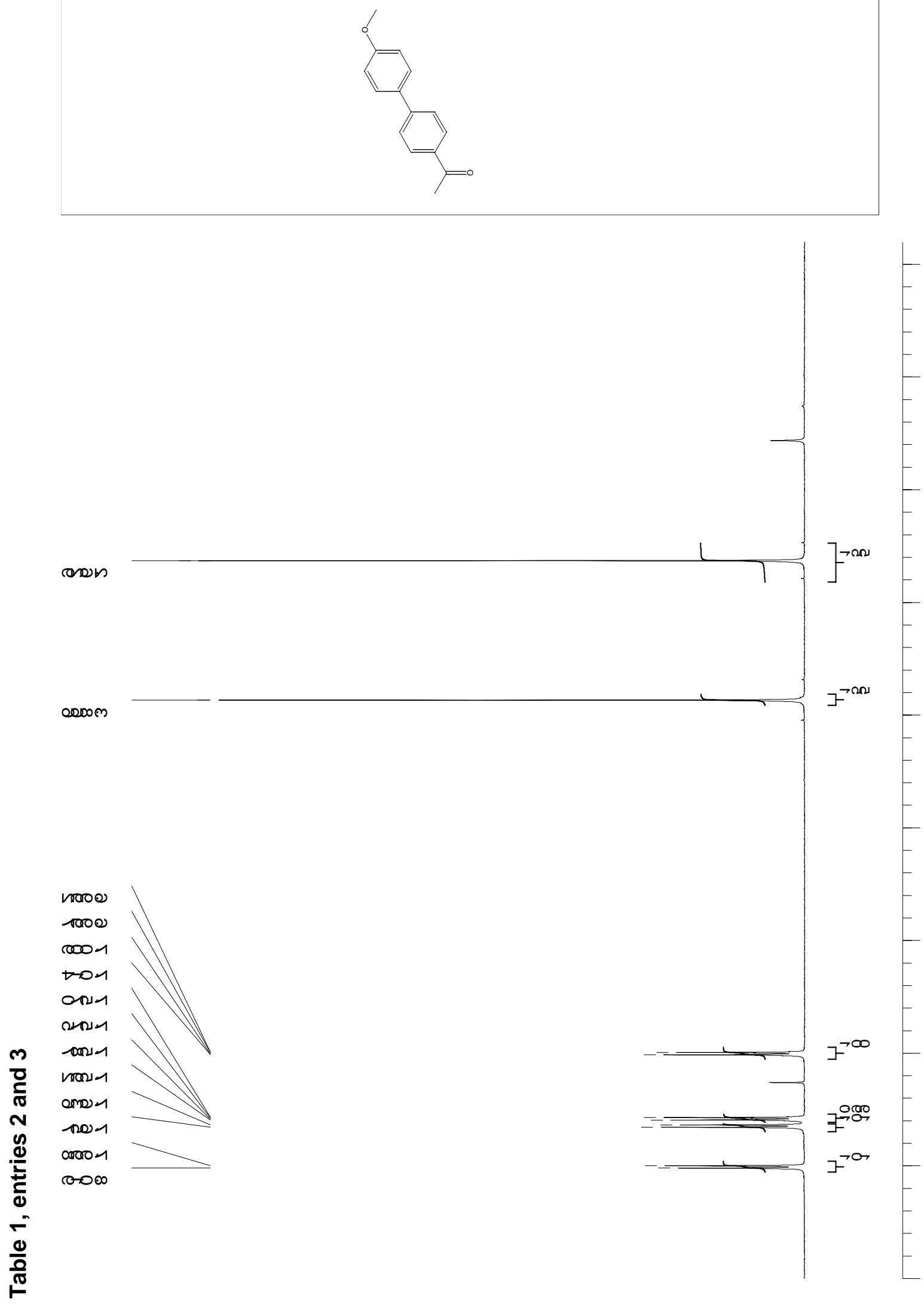


$$
1
$$




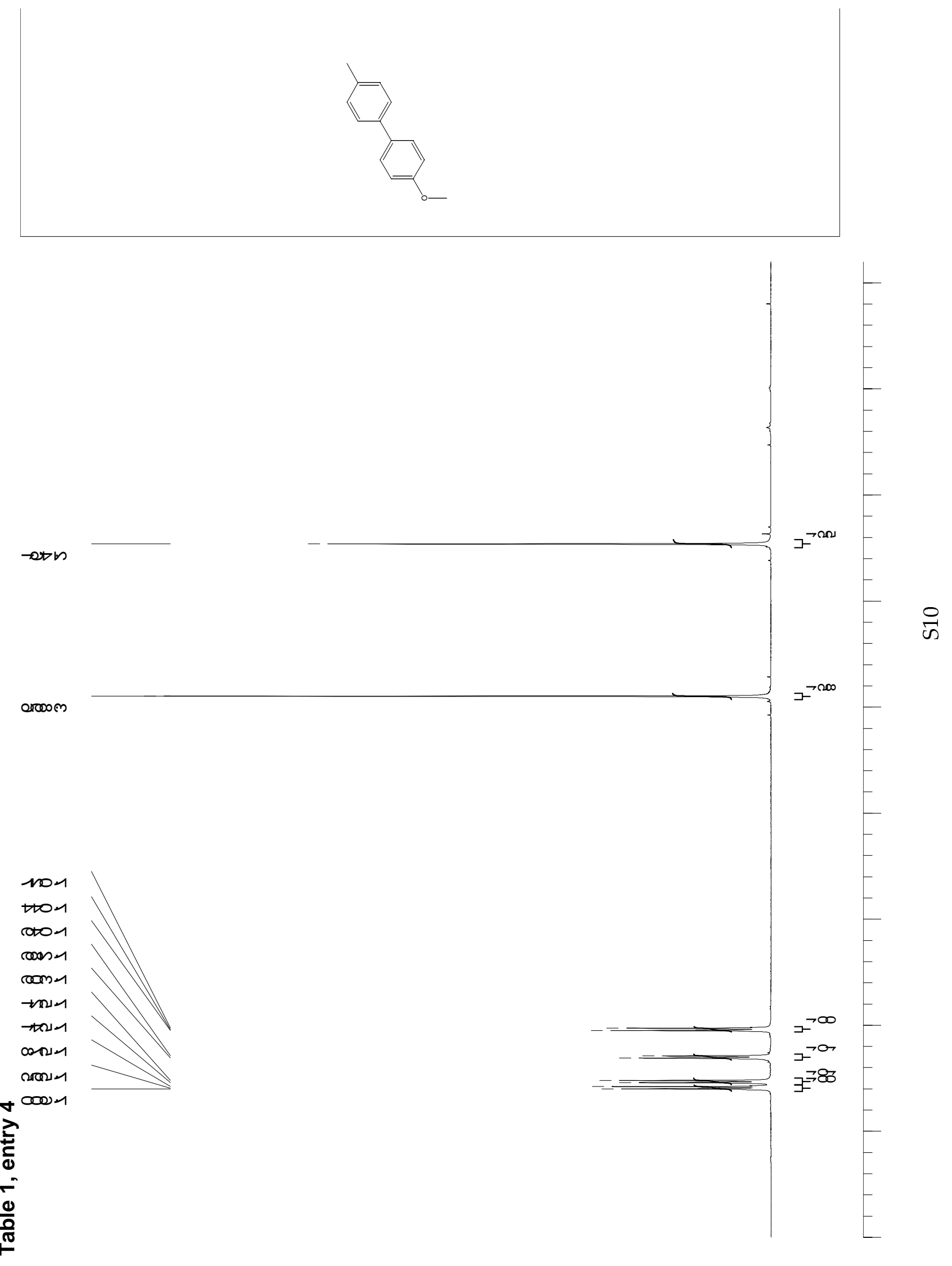



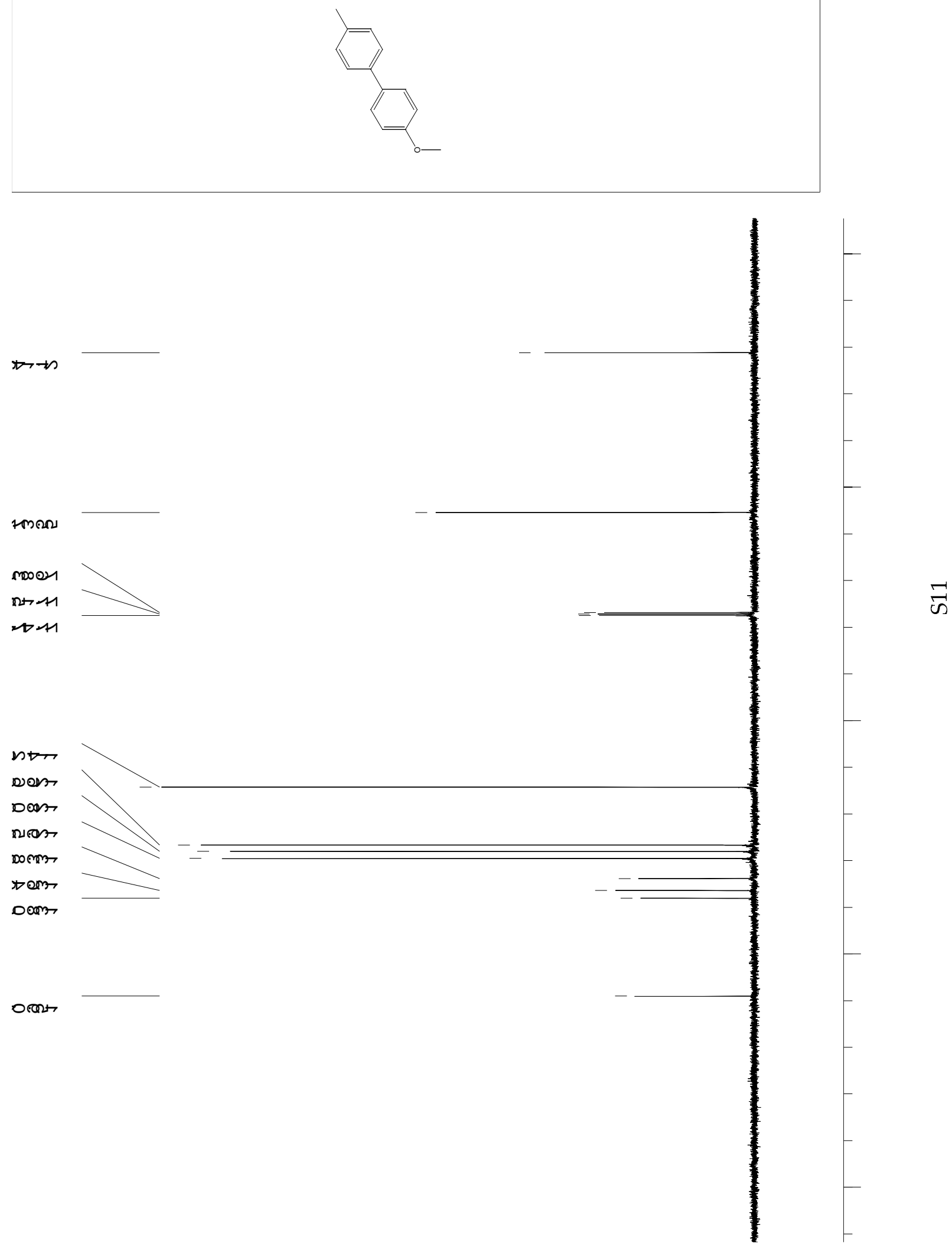

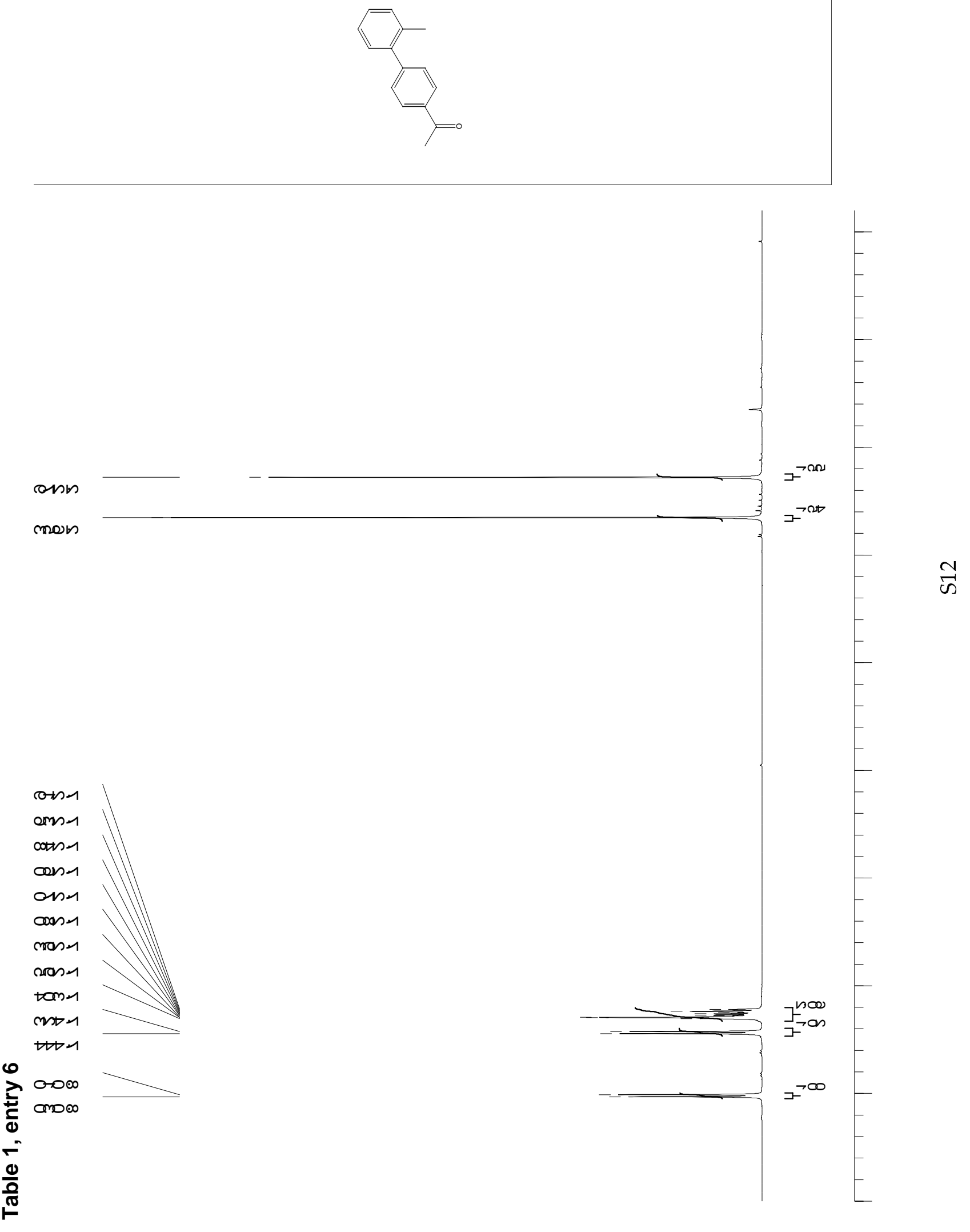


$$
1
$$




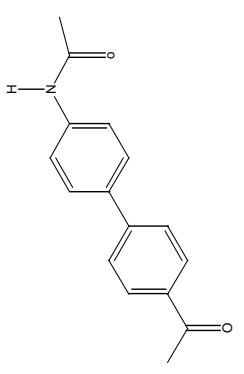

mon

CONLS

ramm

sor

क्मा1

cosis

corol

$-\infty$
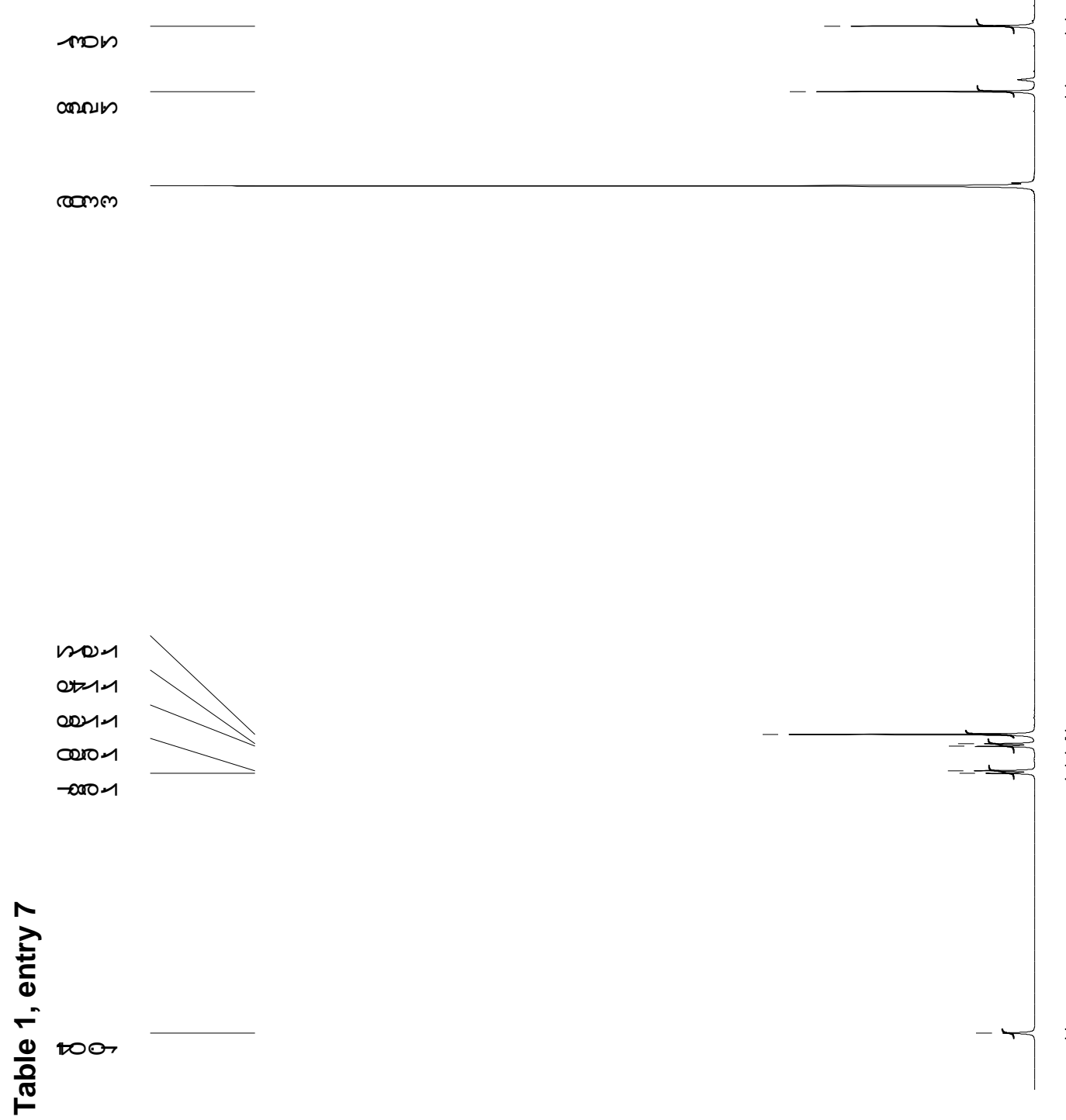

]$^{\text {mess }}$

$\beth^{m-1}$

$\frac{ \pm}{0}$ 


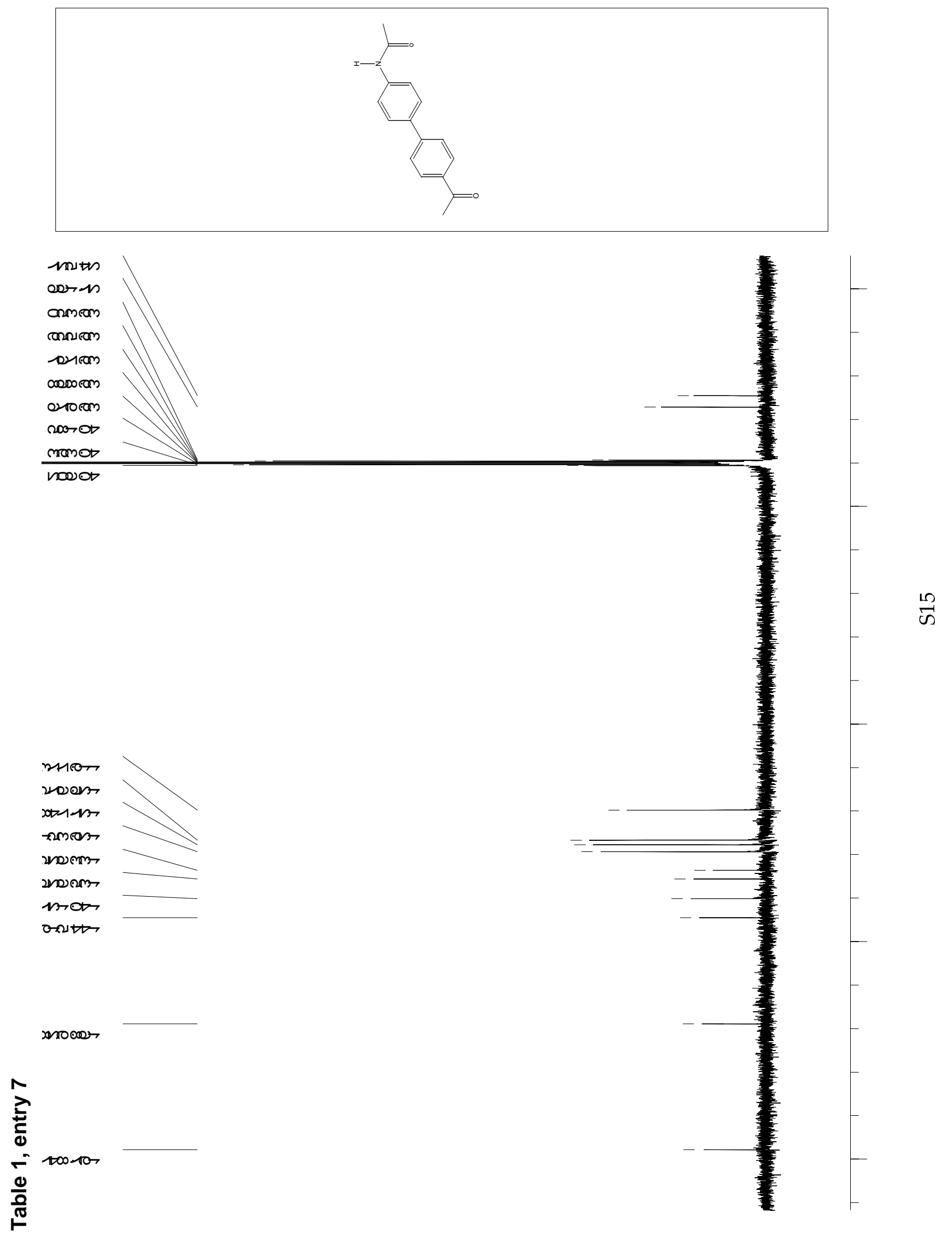




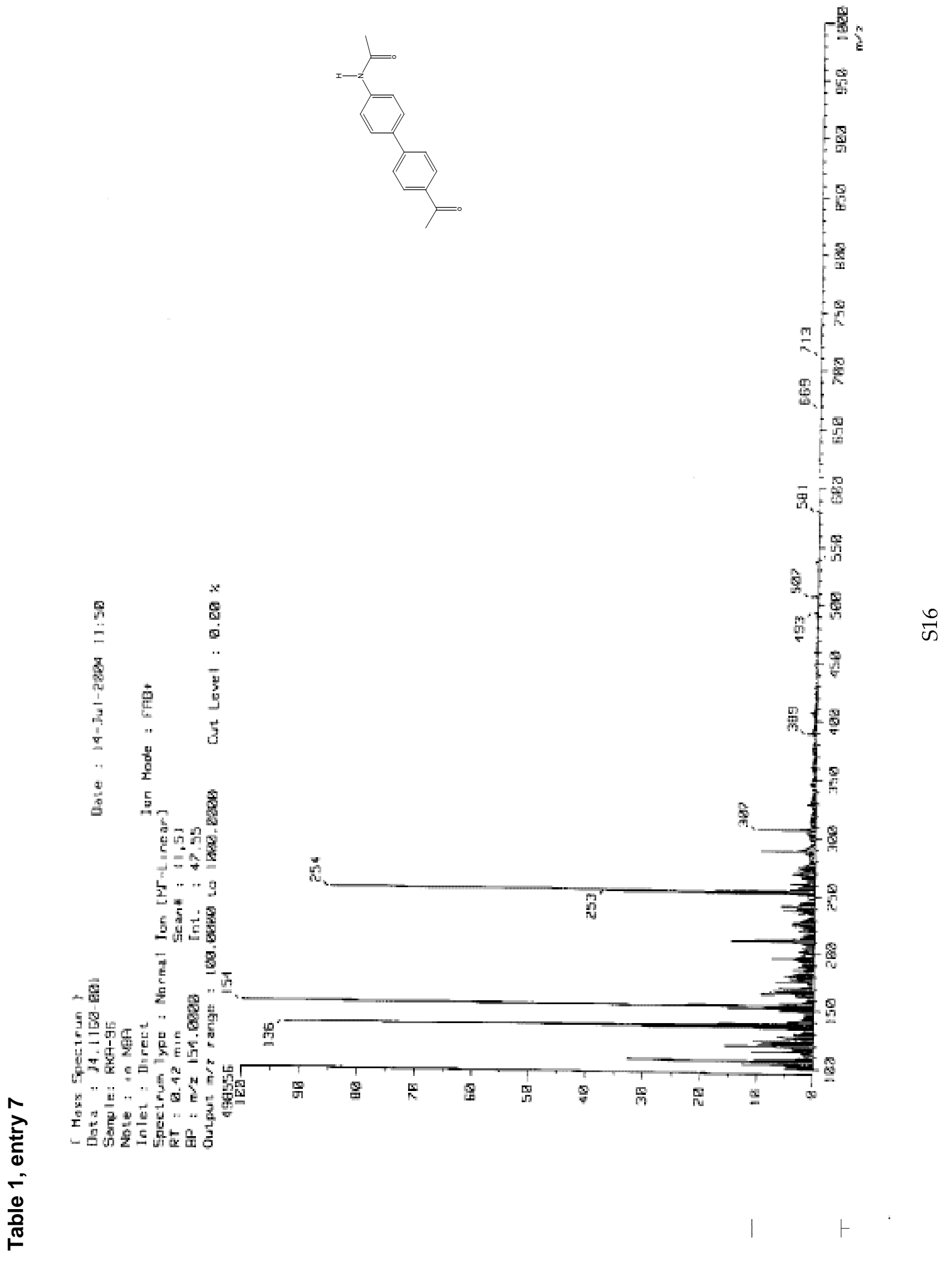




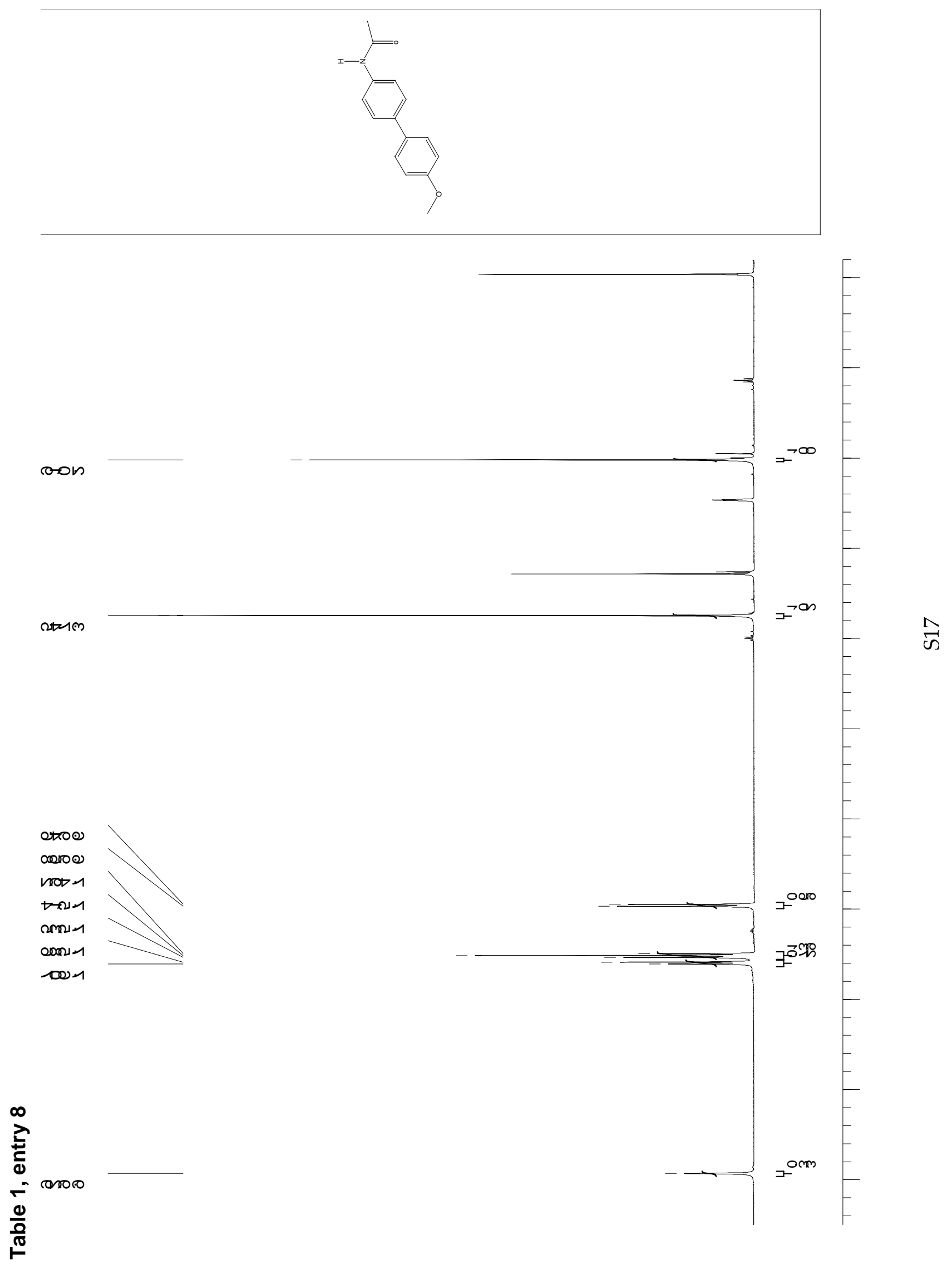



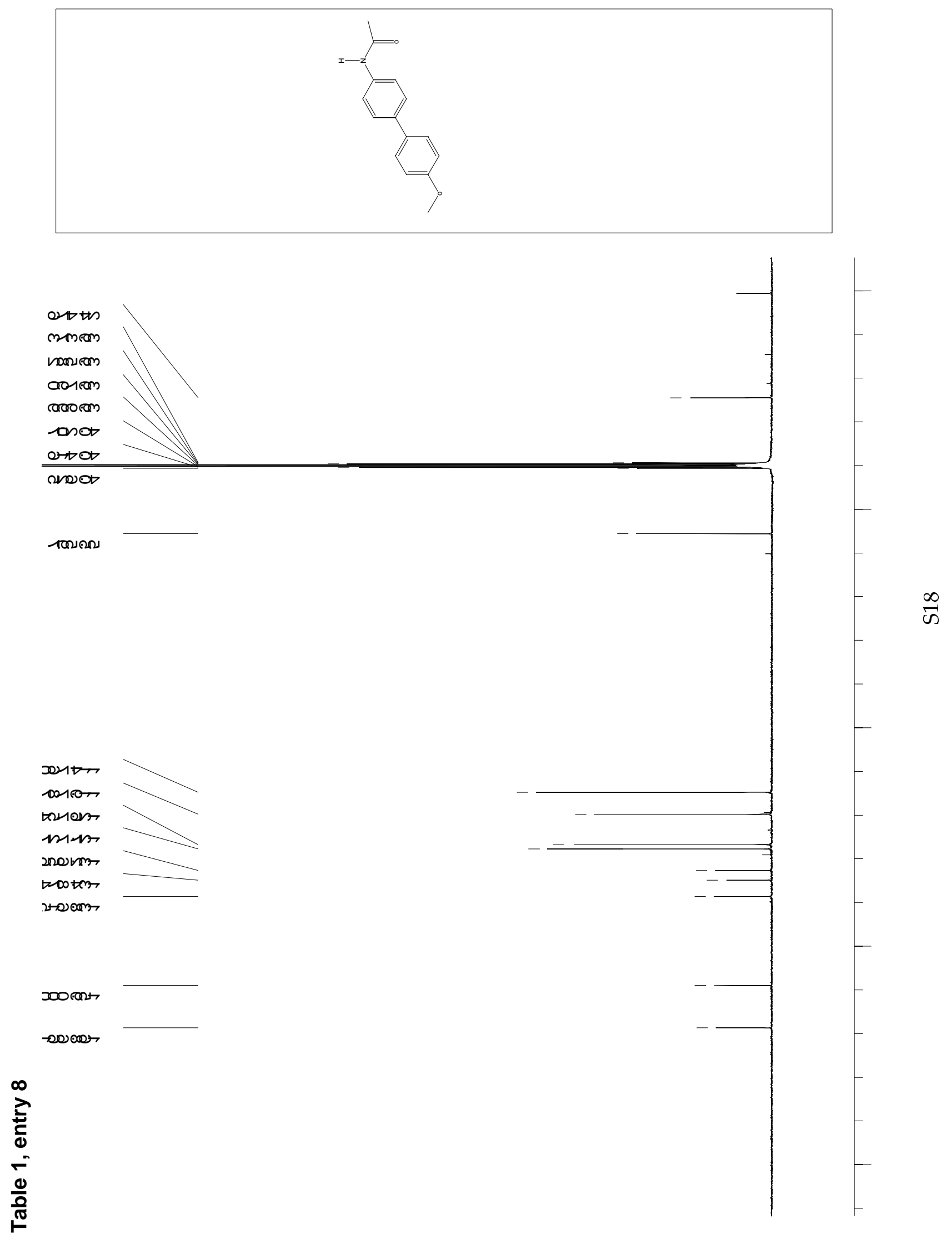

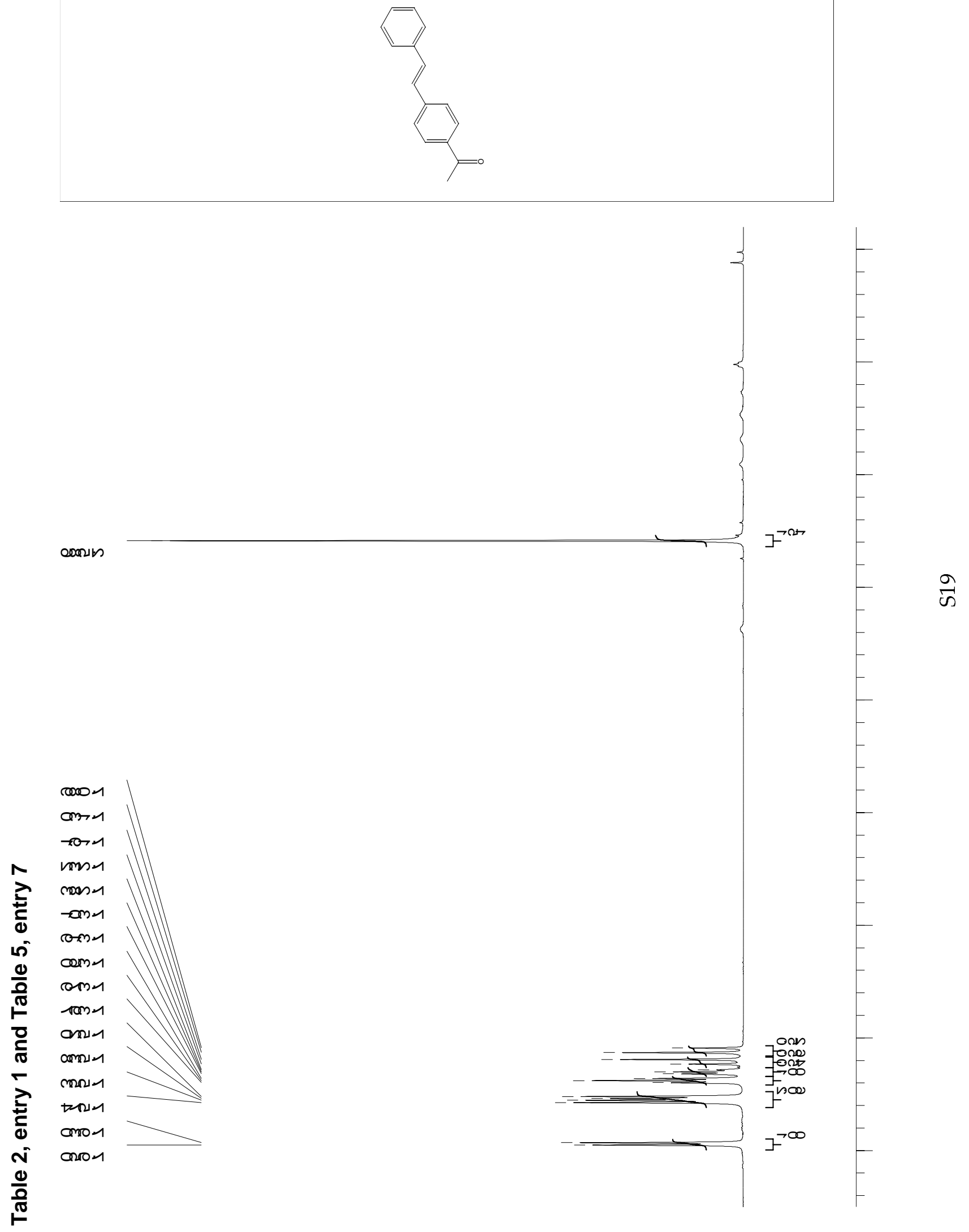

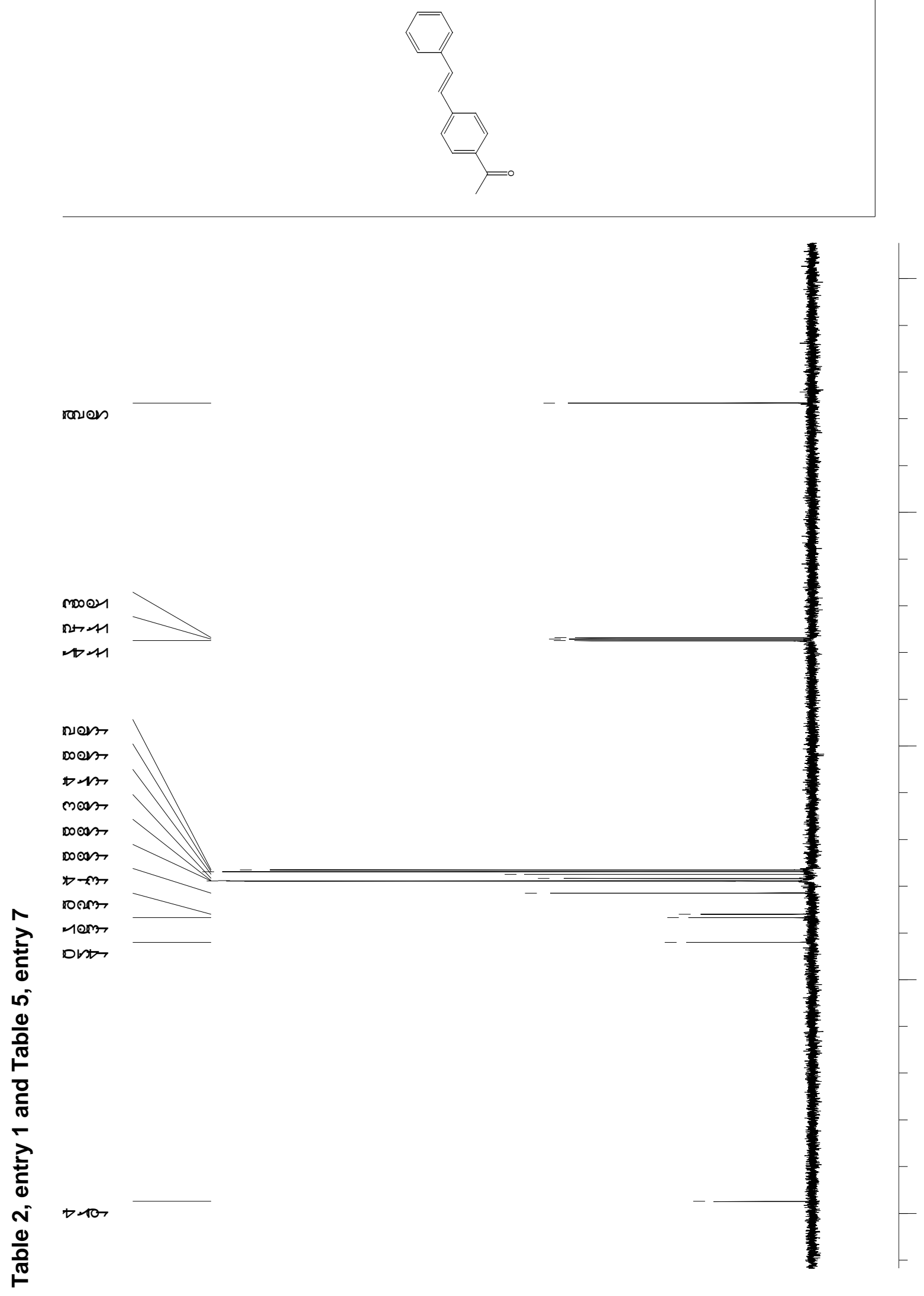

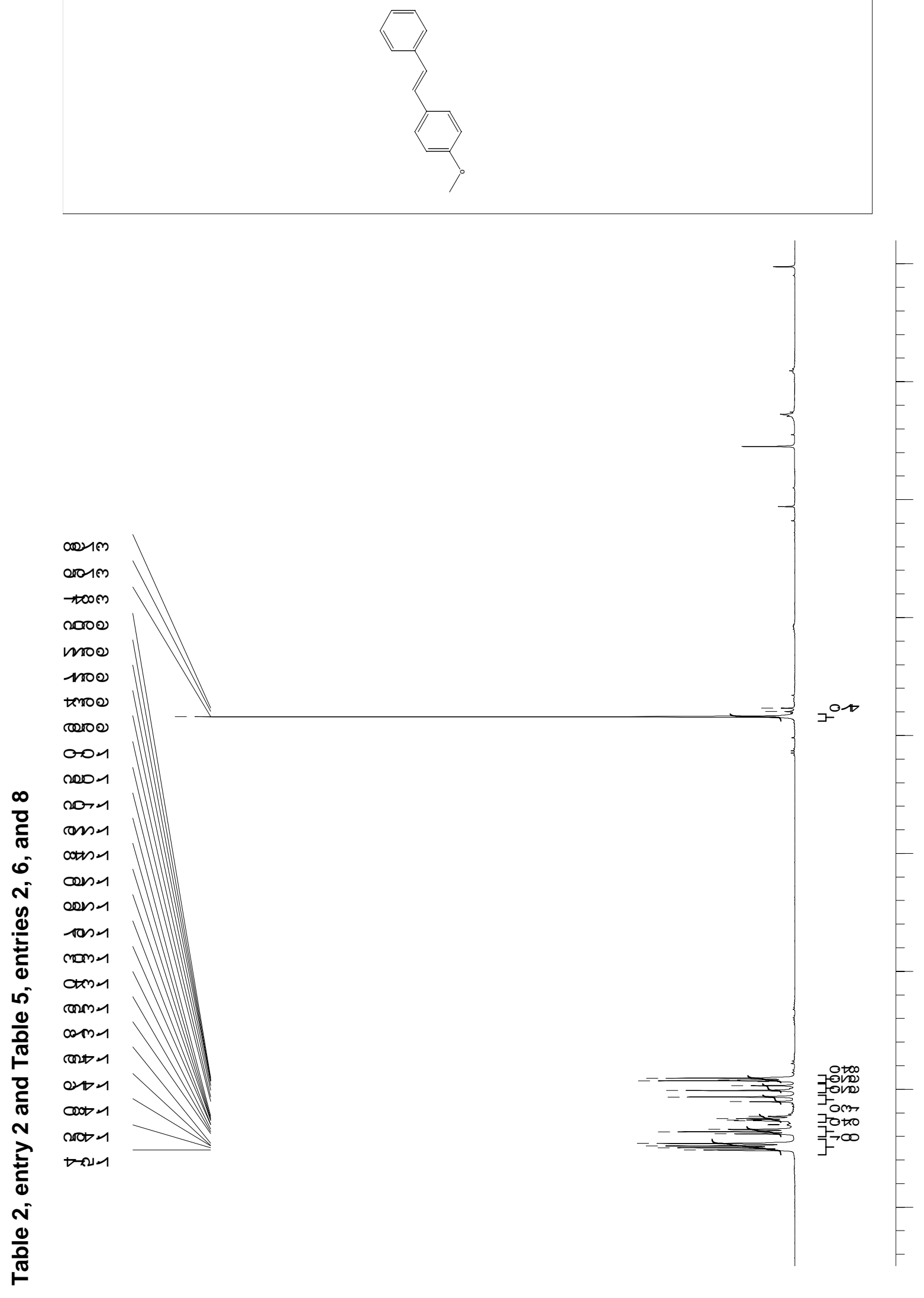


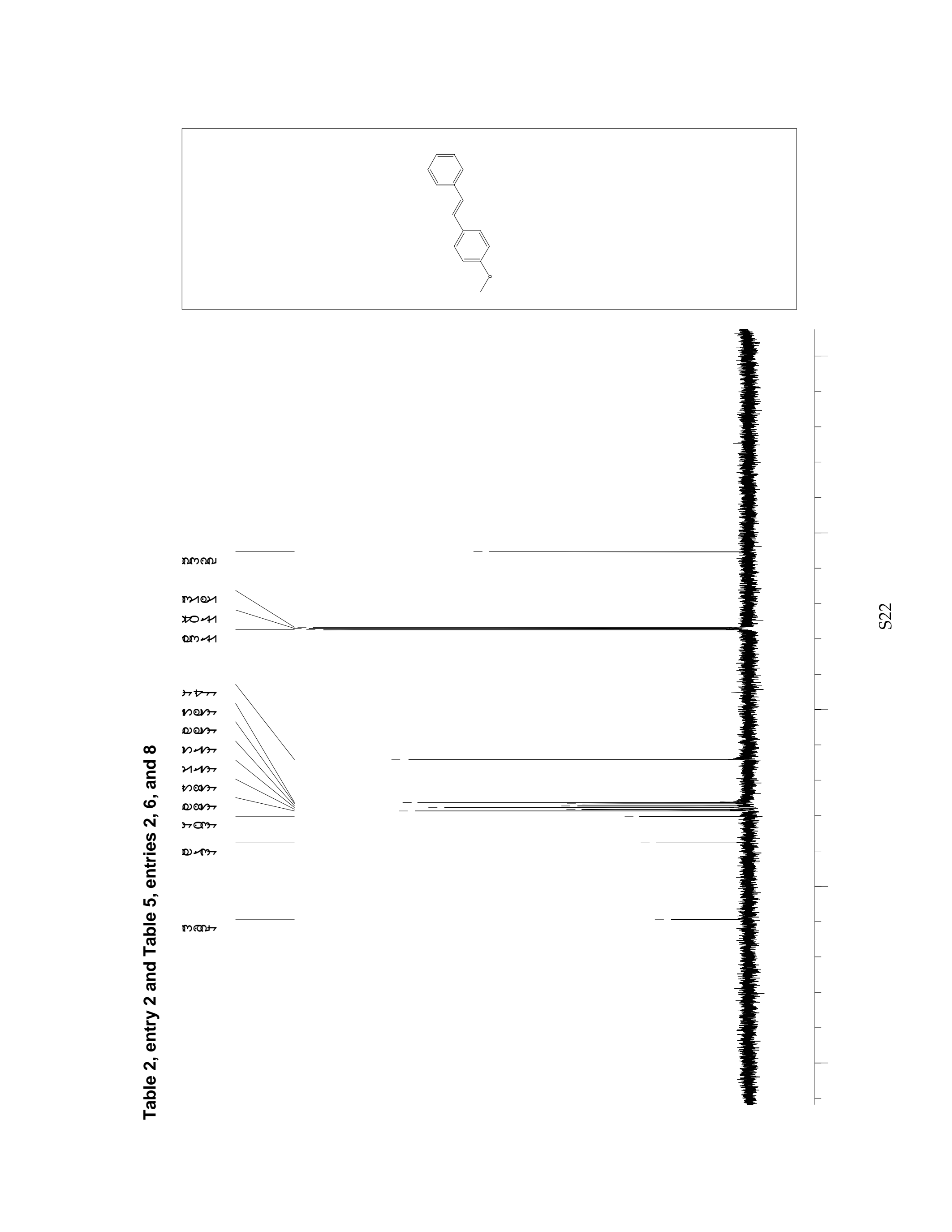



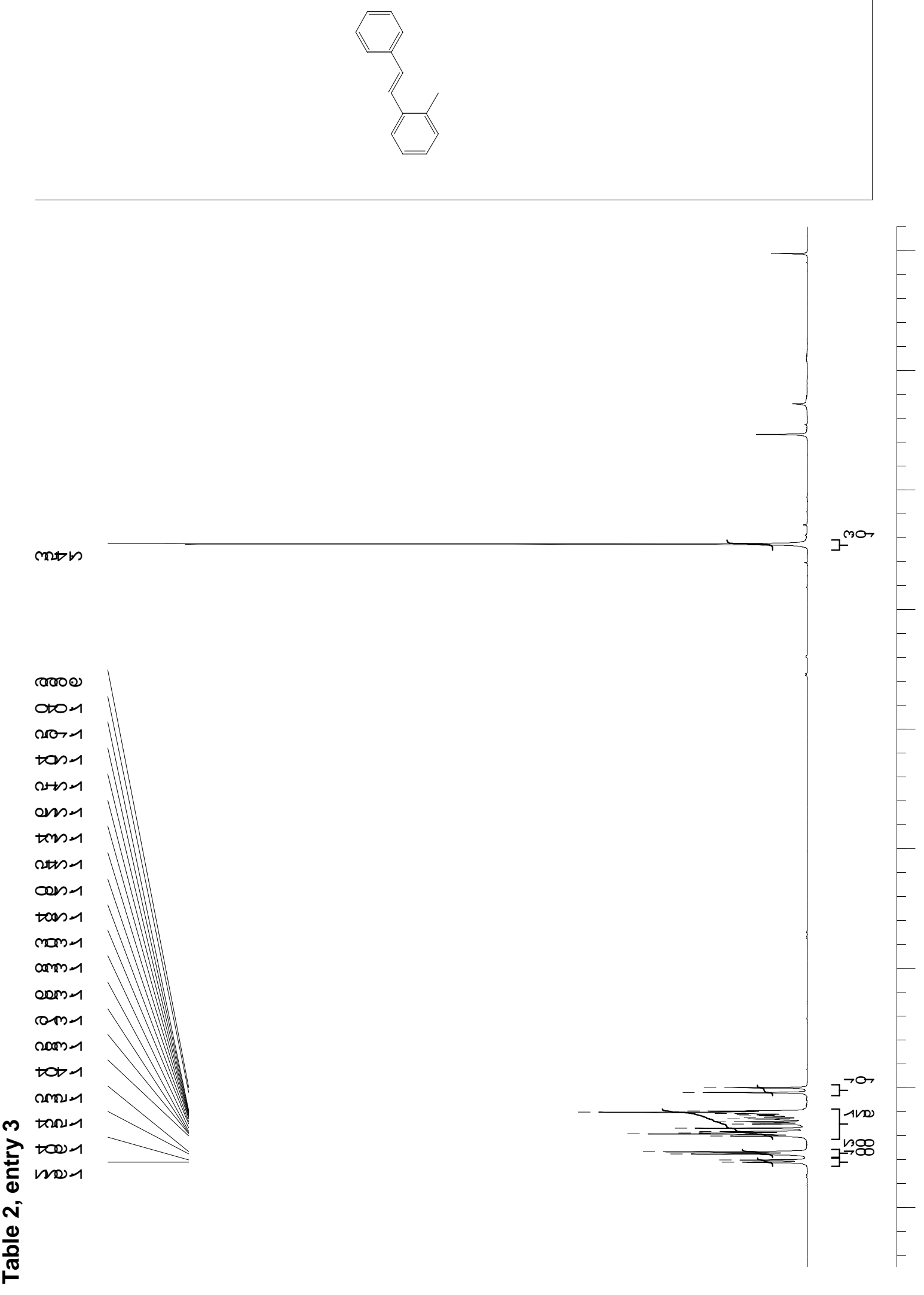


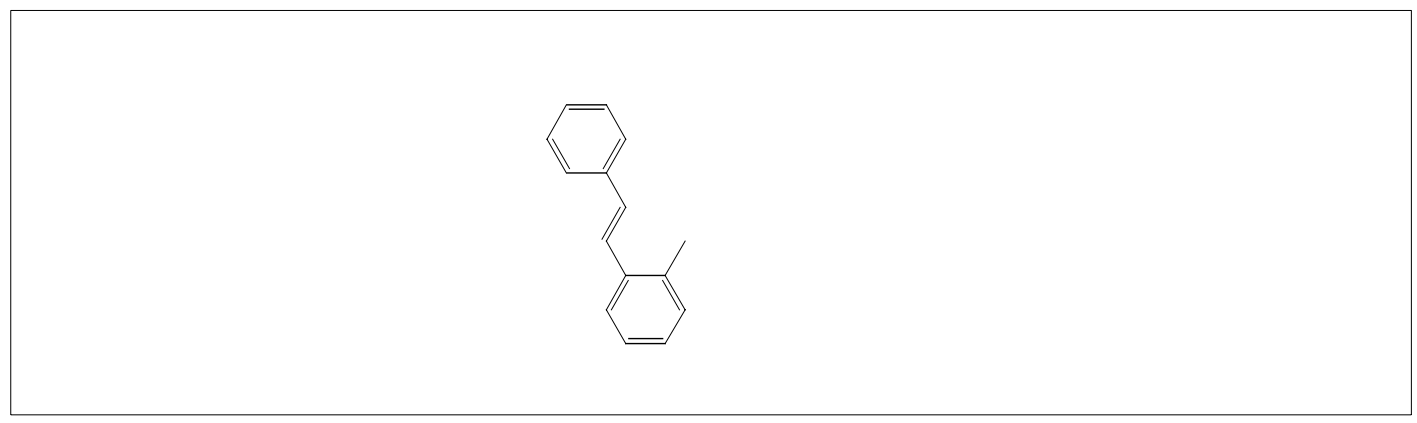

श्वा०

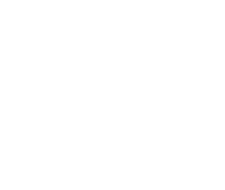

sher

to 11

Don 11

דथल

מलD

NQ⿻

प्य

דעים

yoos

Dबr

tar

गलख्य

गब्य

यमr

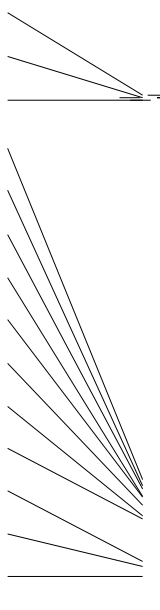

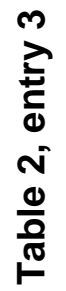



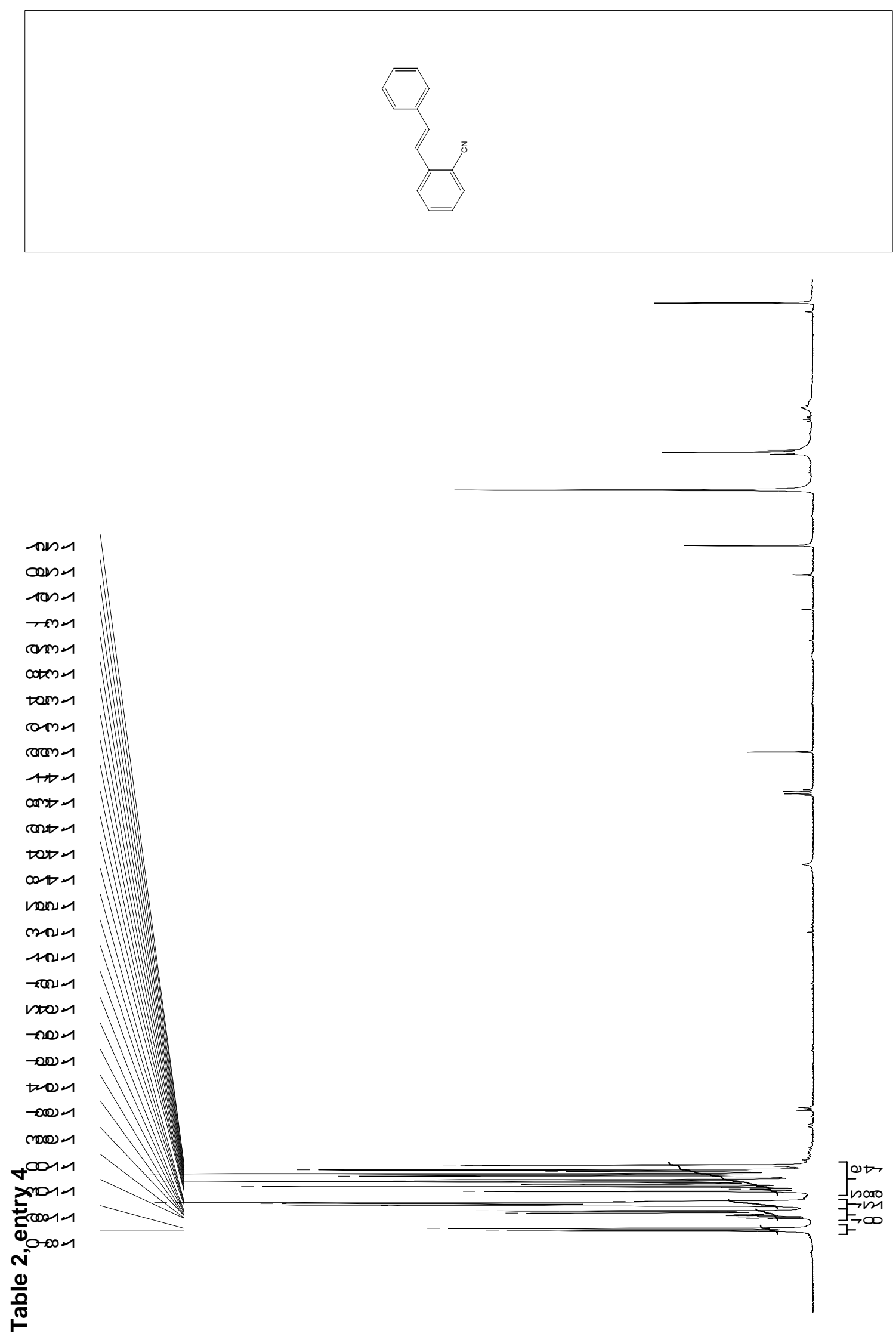

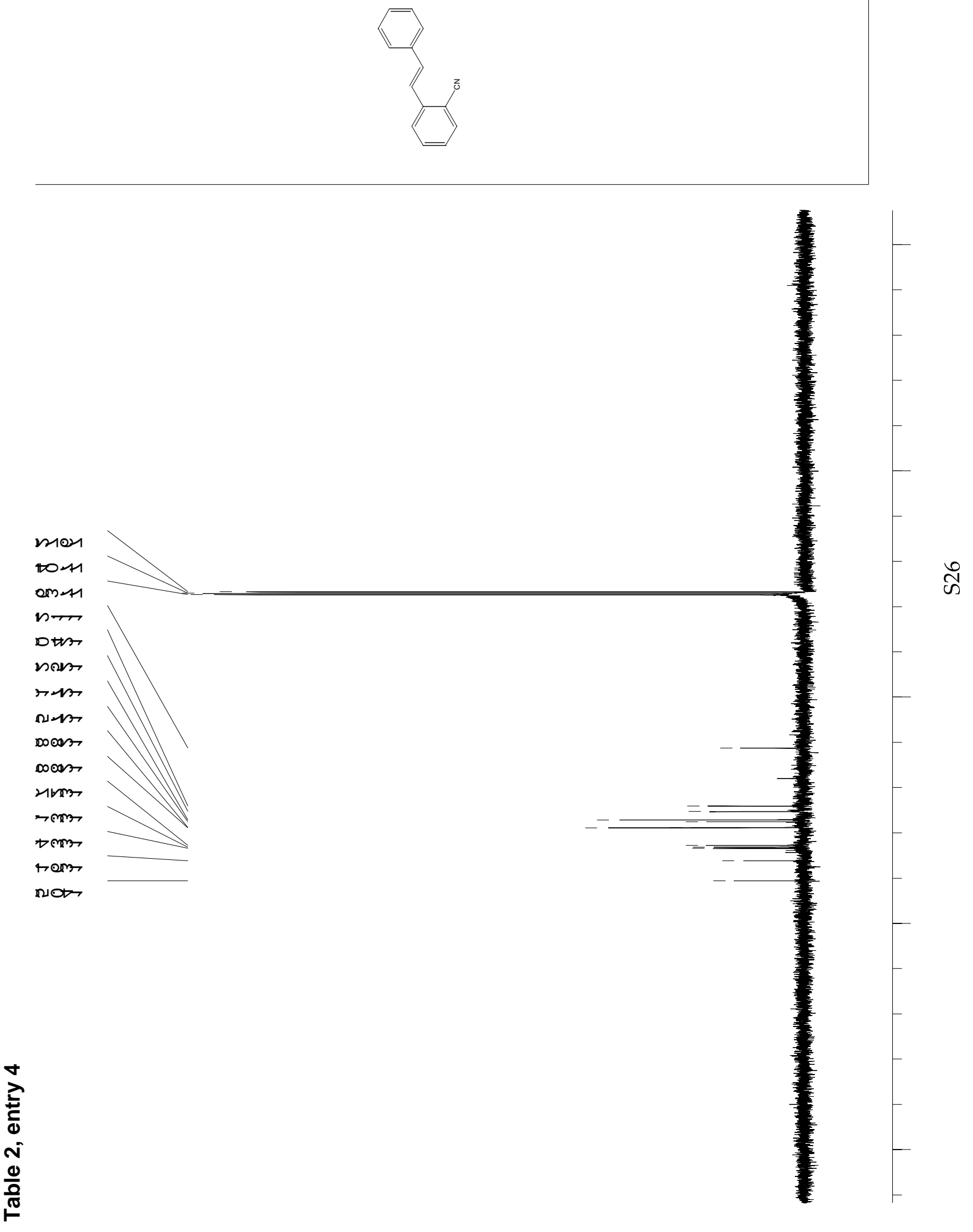

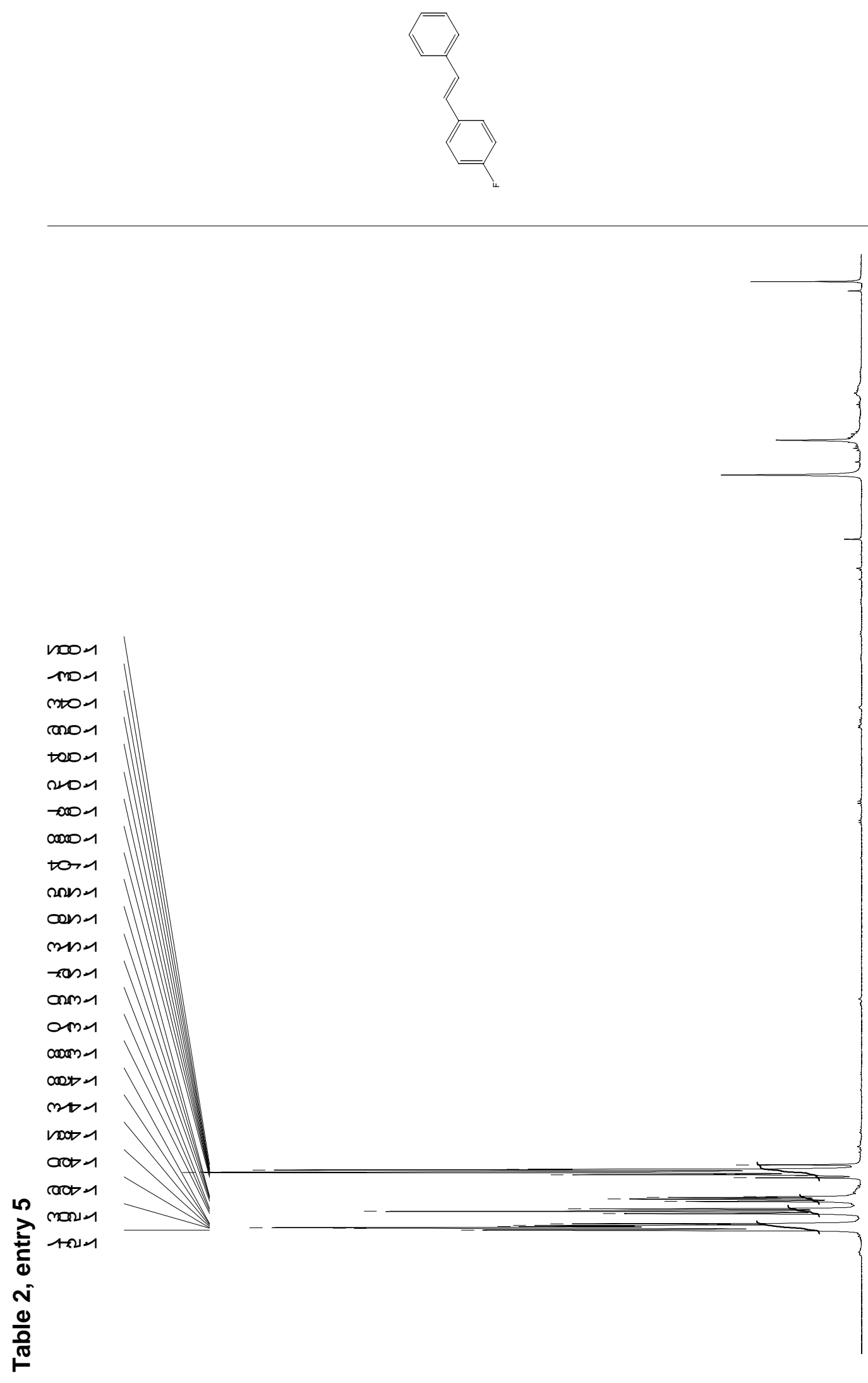


$$
A
$$




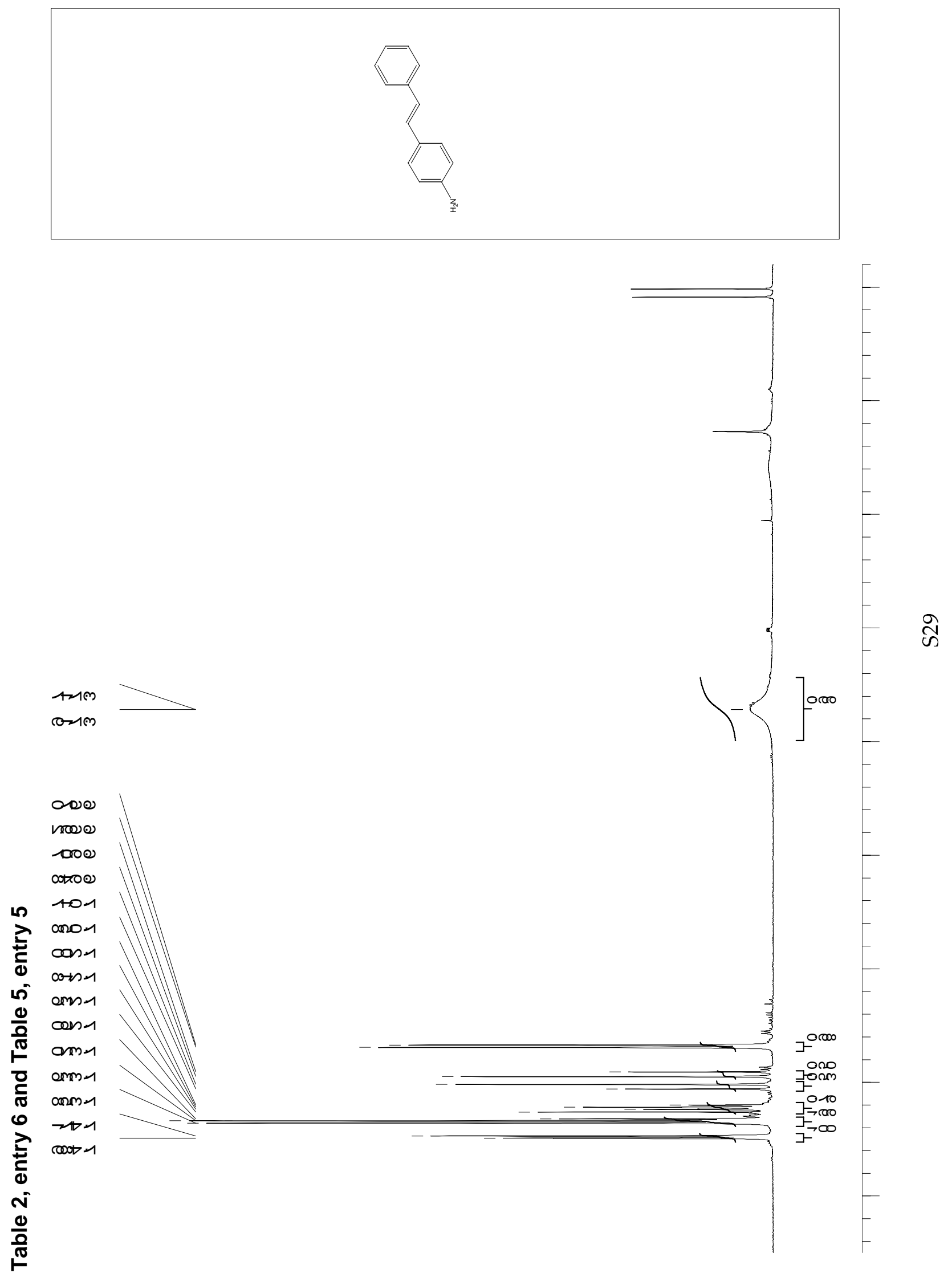



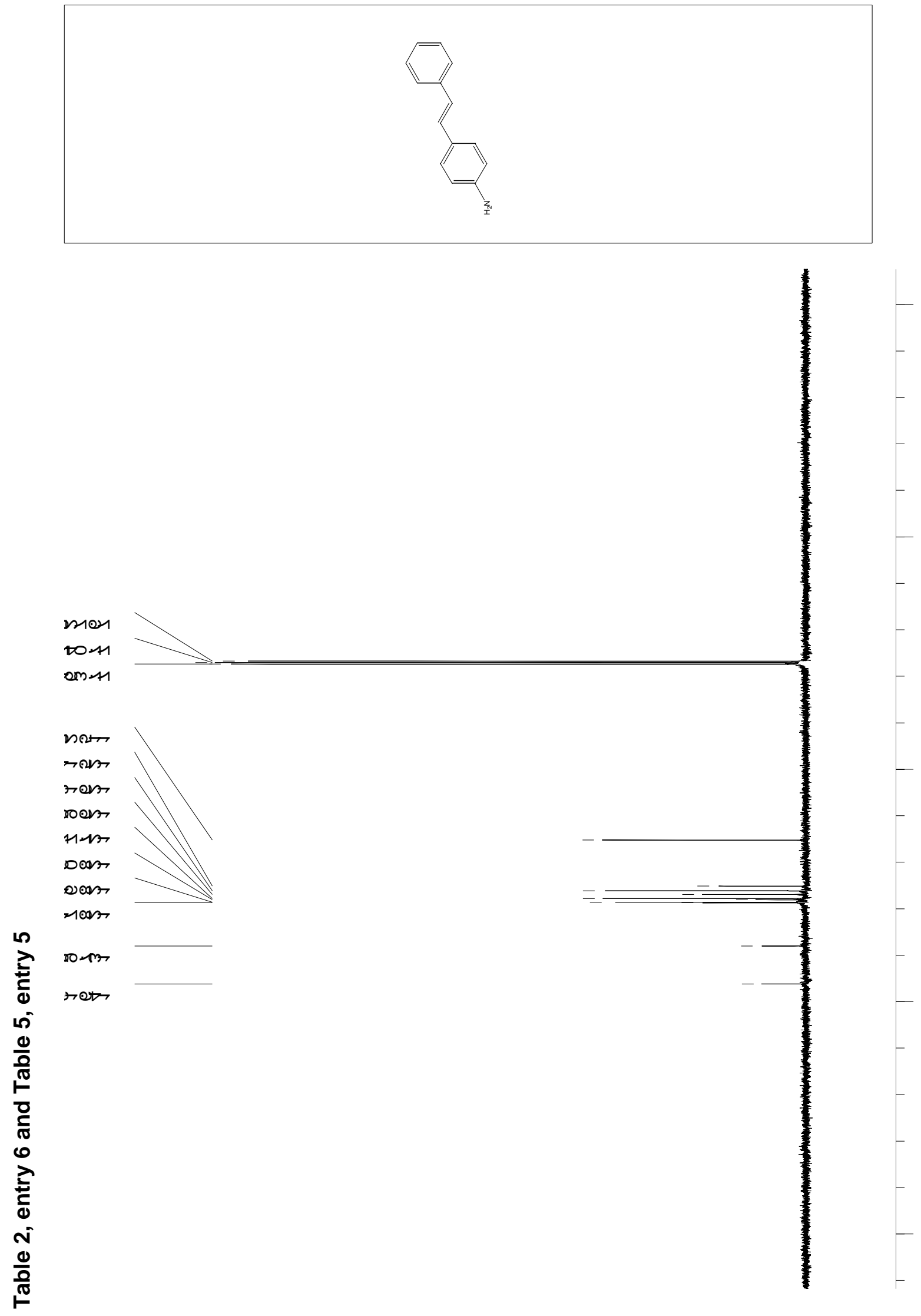


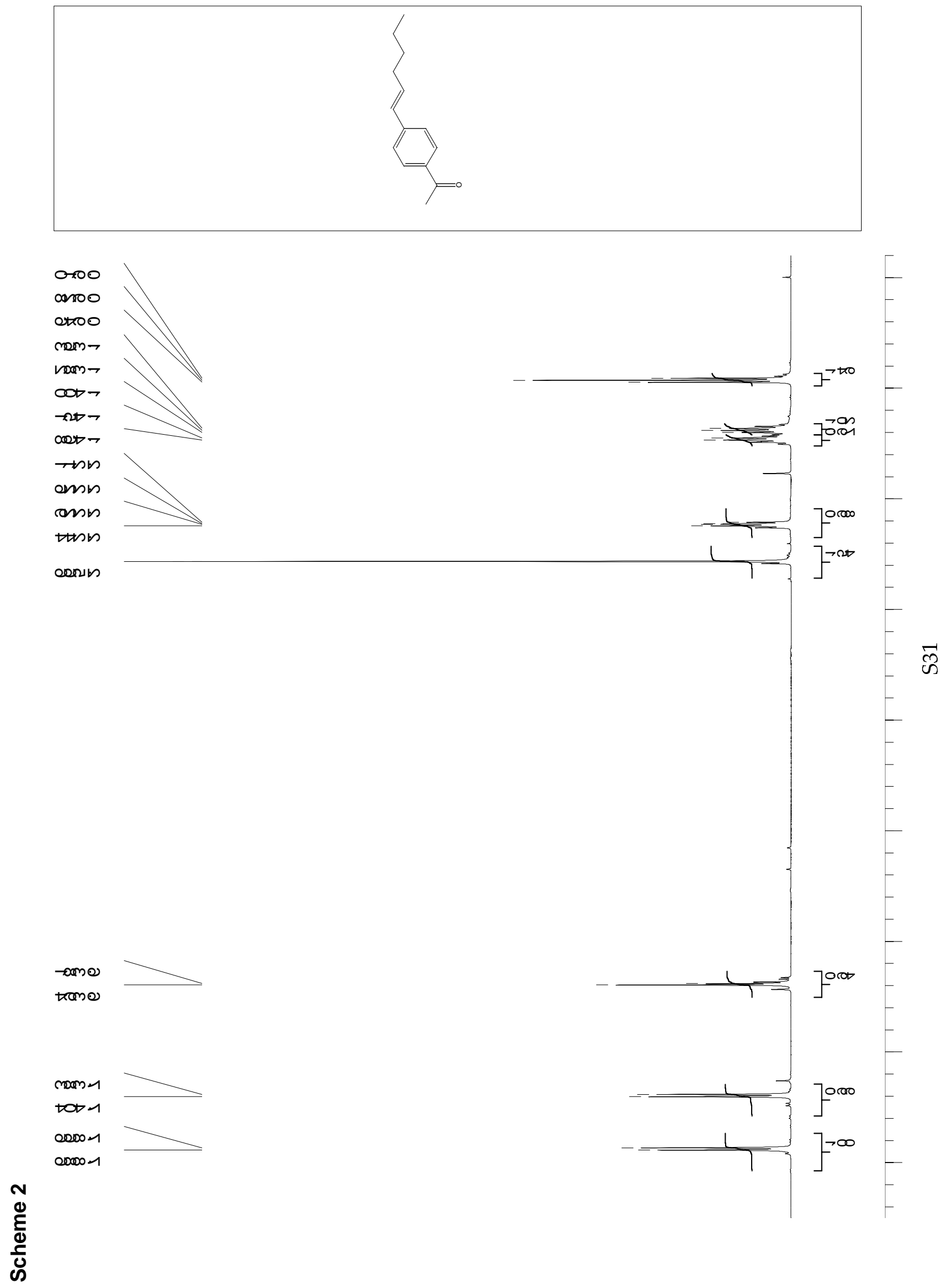




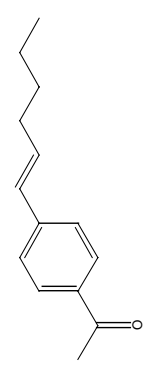

trom

rosts

स्थलes

cos-m

nootrom

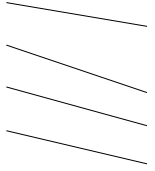

nuas

cos

Don

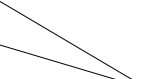

प्वस्य

roas

roost

ntar

$\triangle \mathrm{DMT}$

Dיד

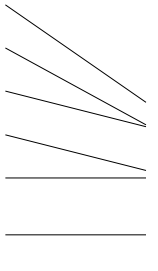

DI

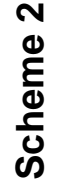

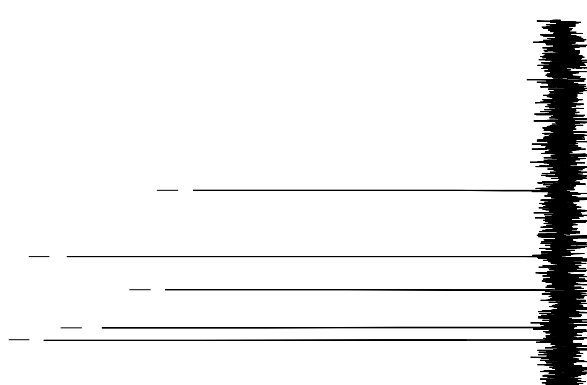




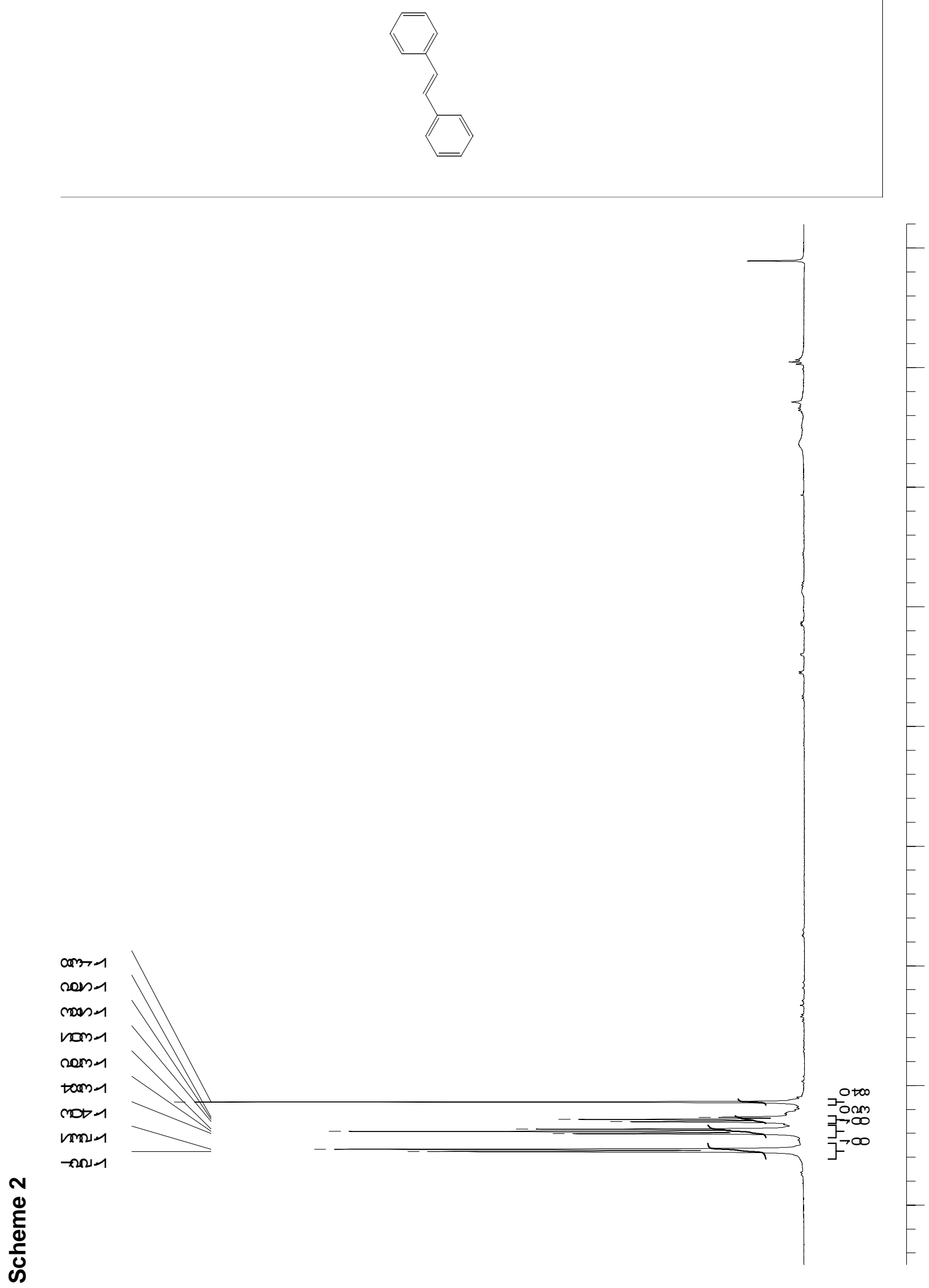




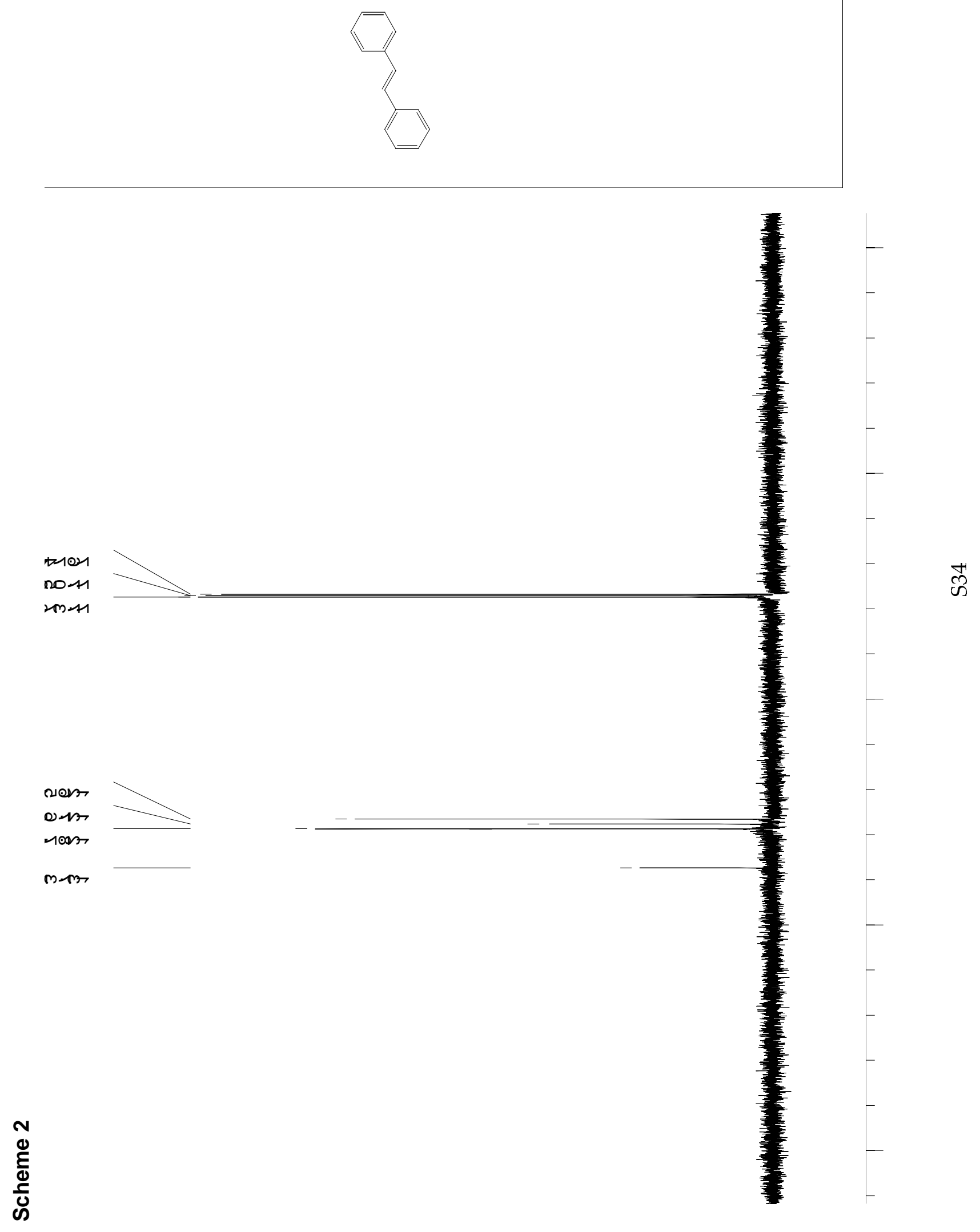



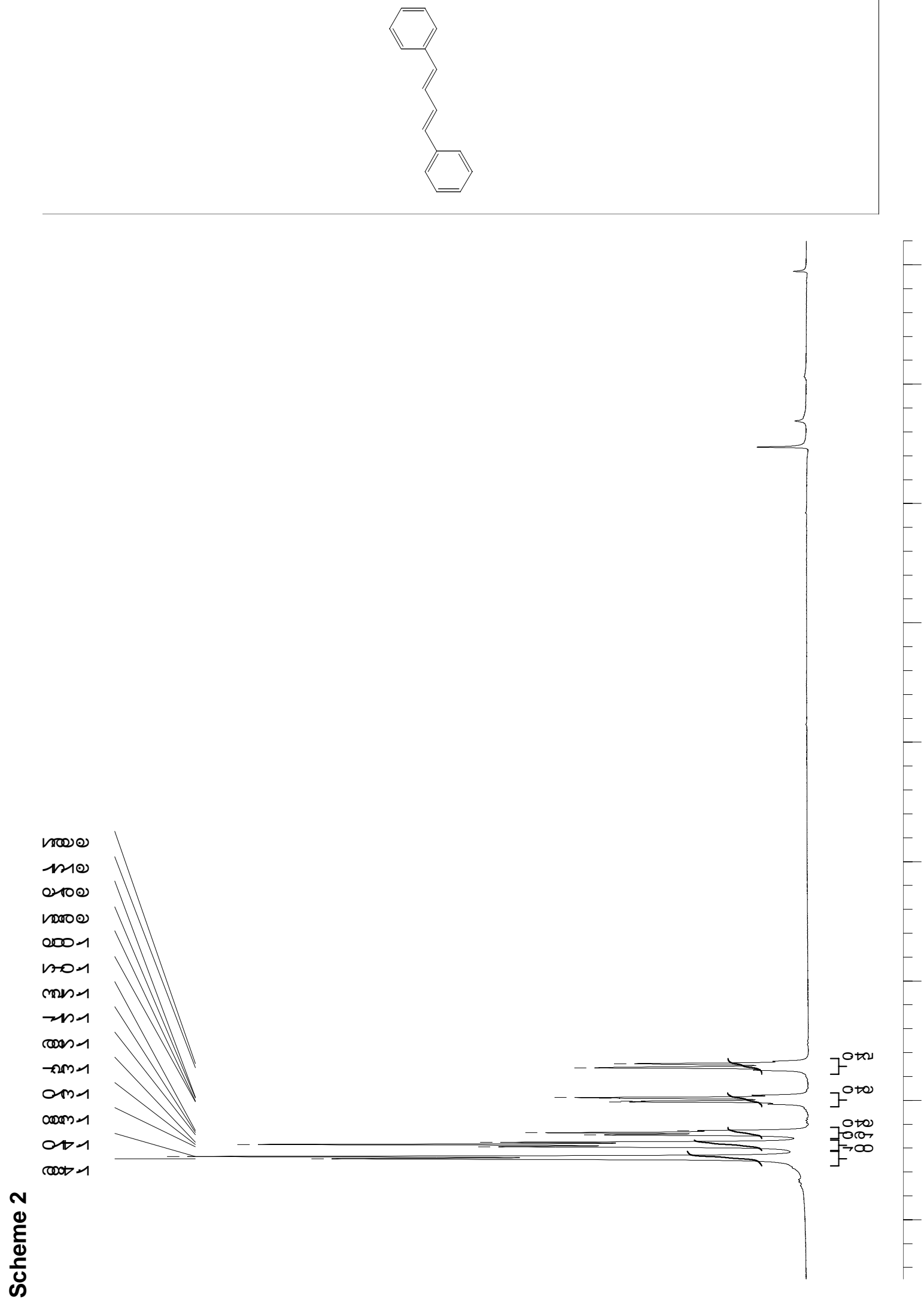

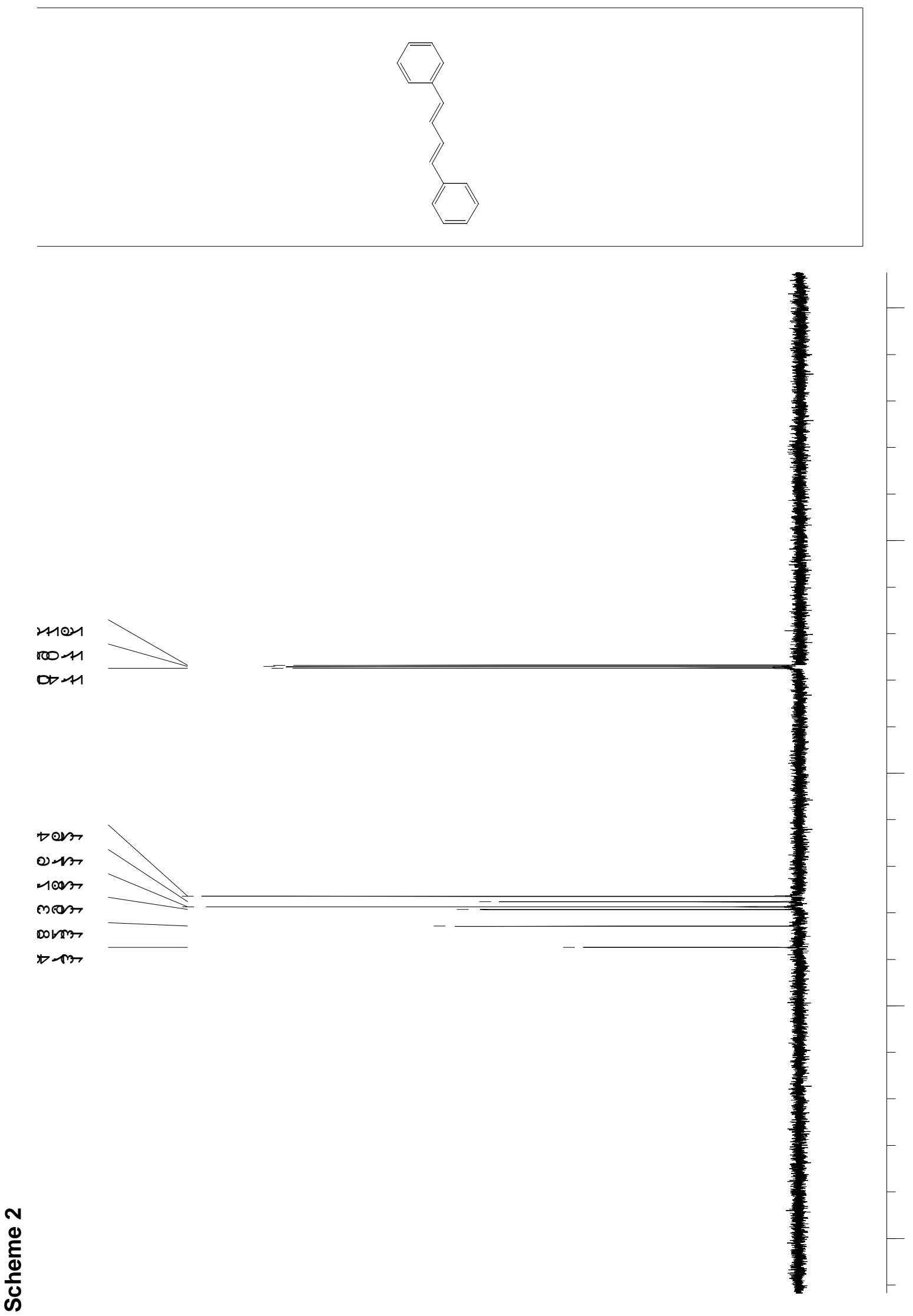

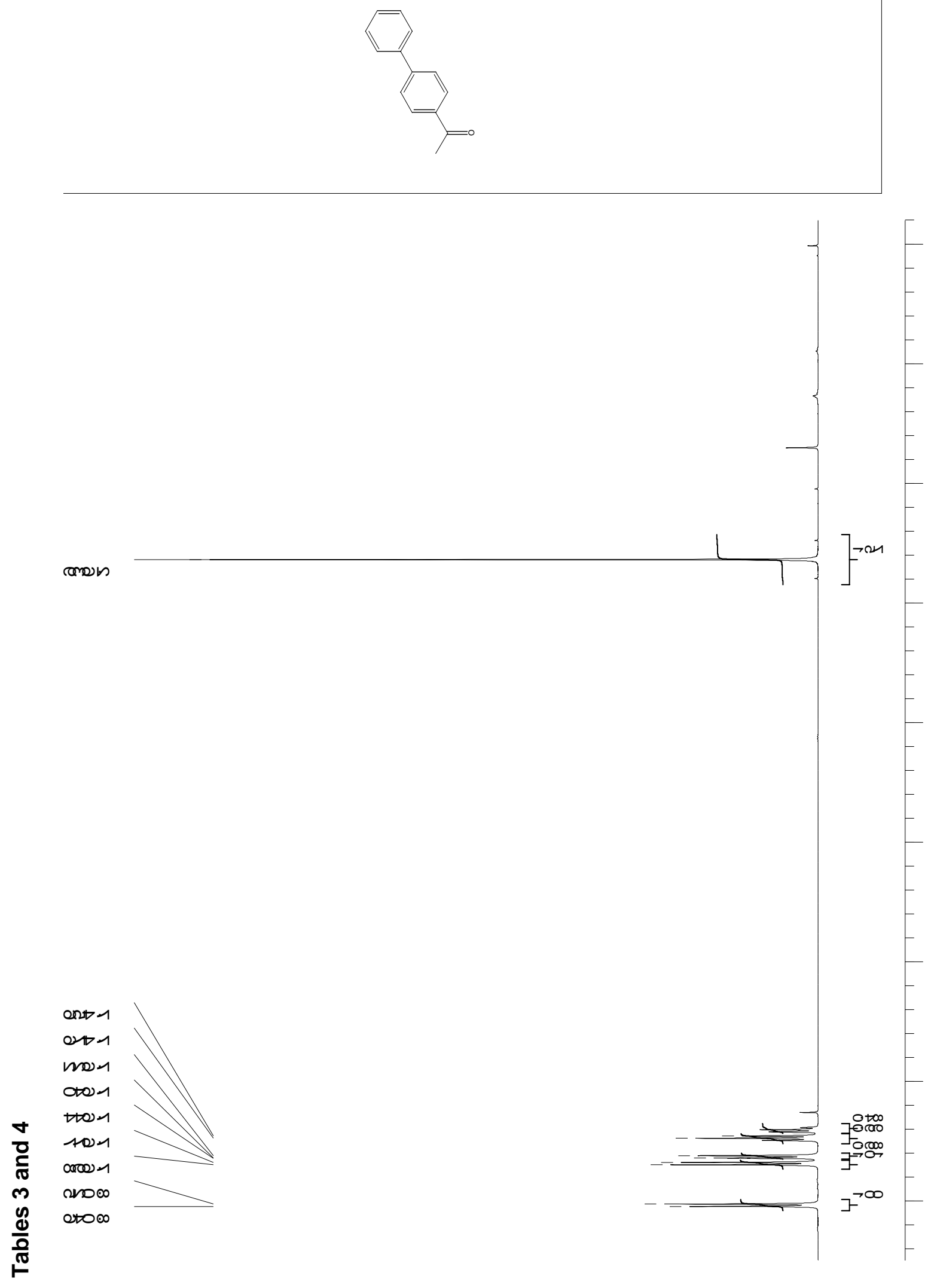


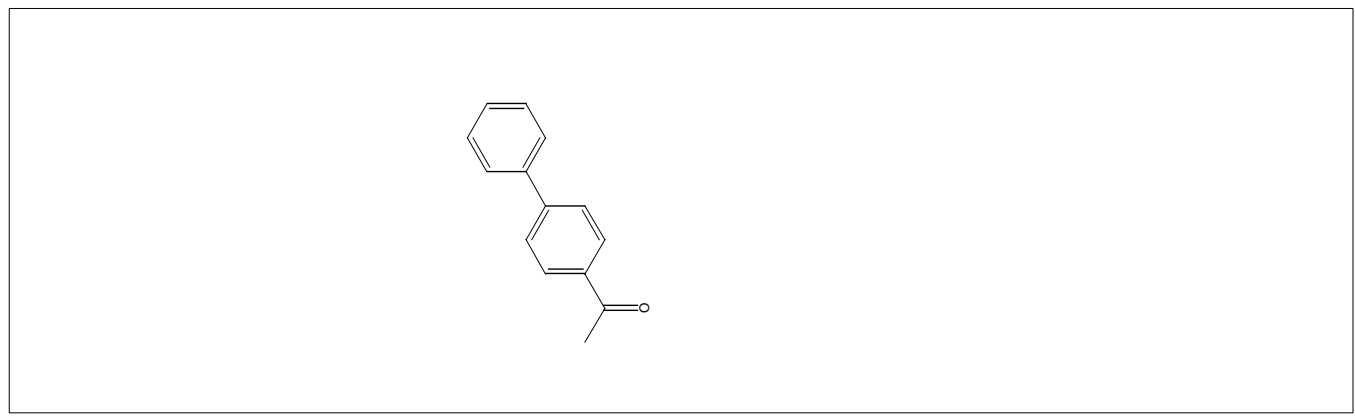

roves

सम्य

2011

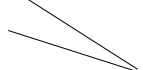

- 1

$=$

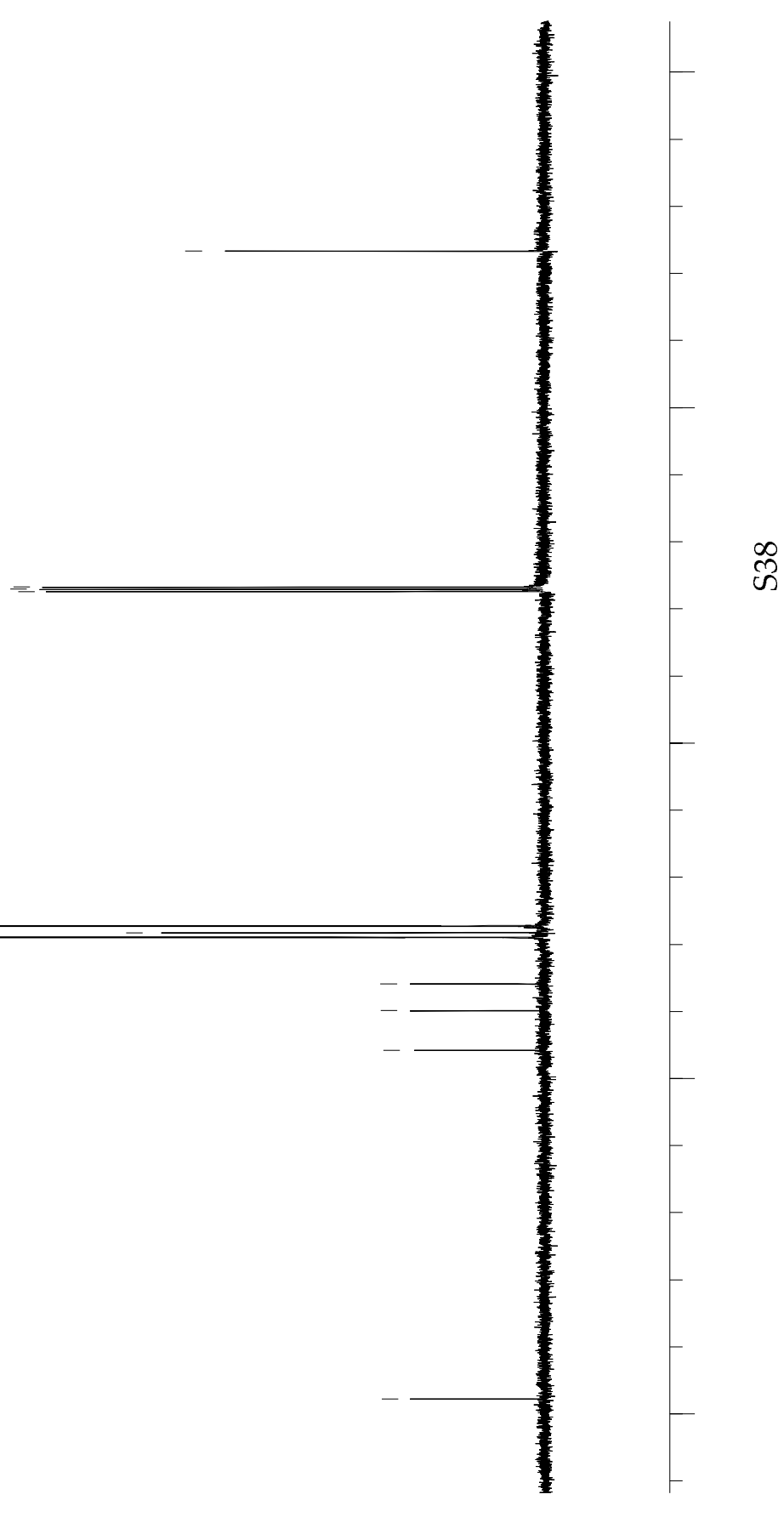

מSAT

חת

noest

roous

rooses

DORM

Dorar

भत्बत 


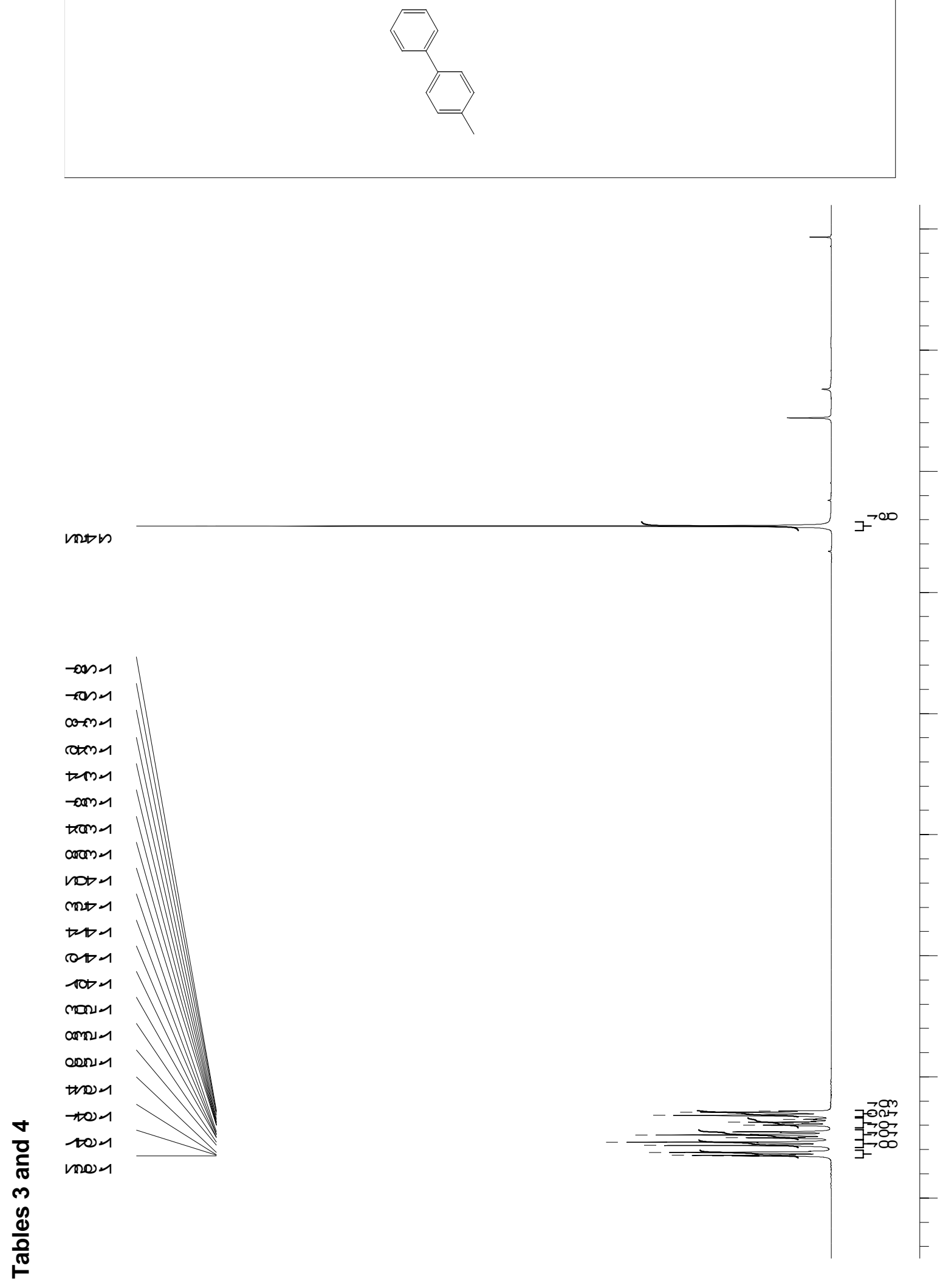




$$
A
$$




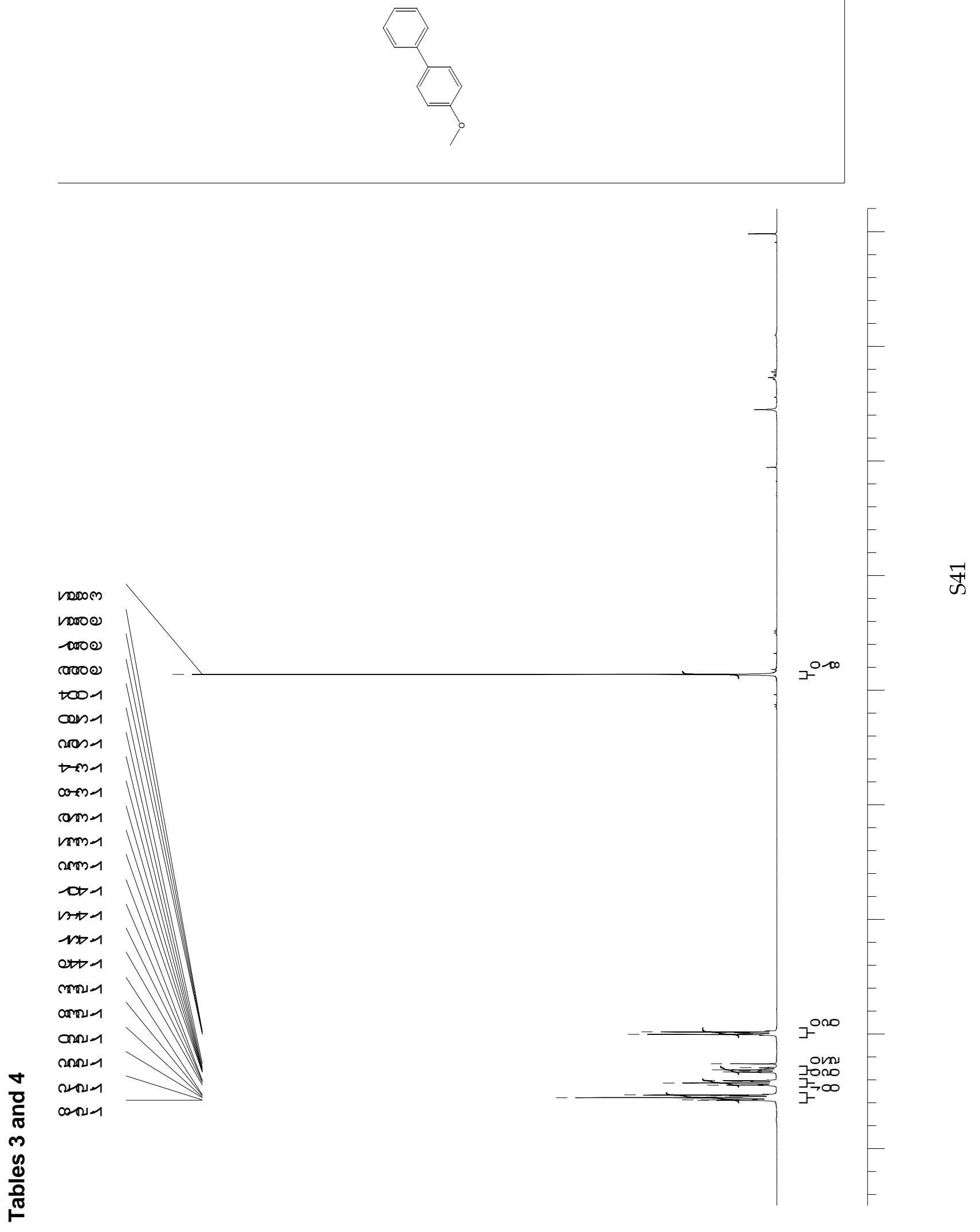



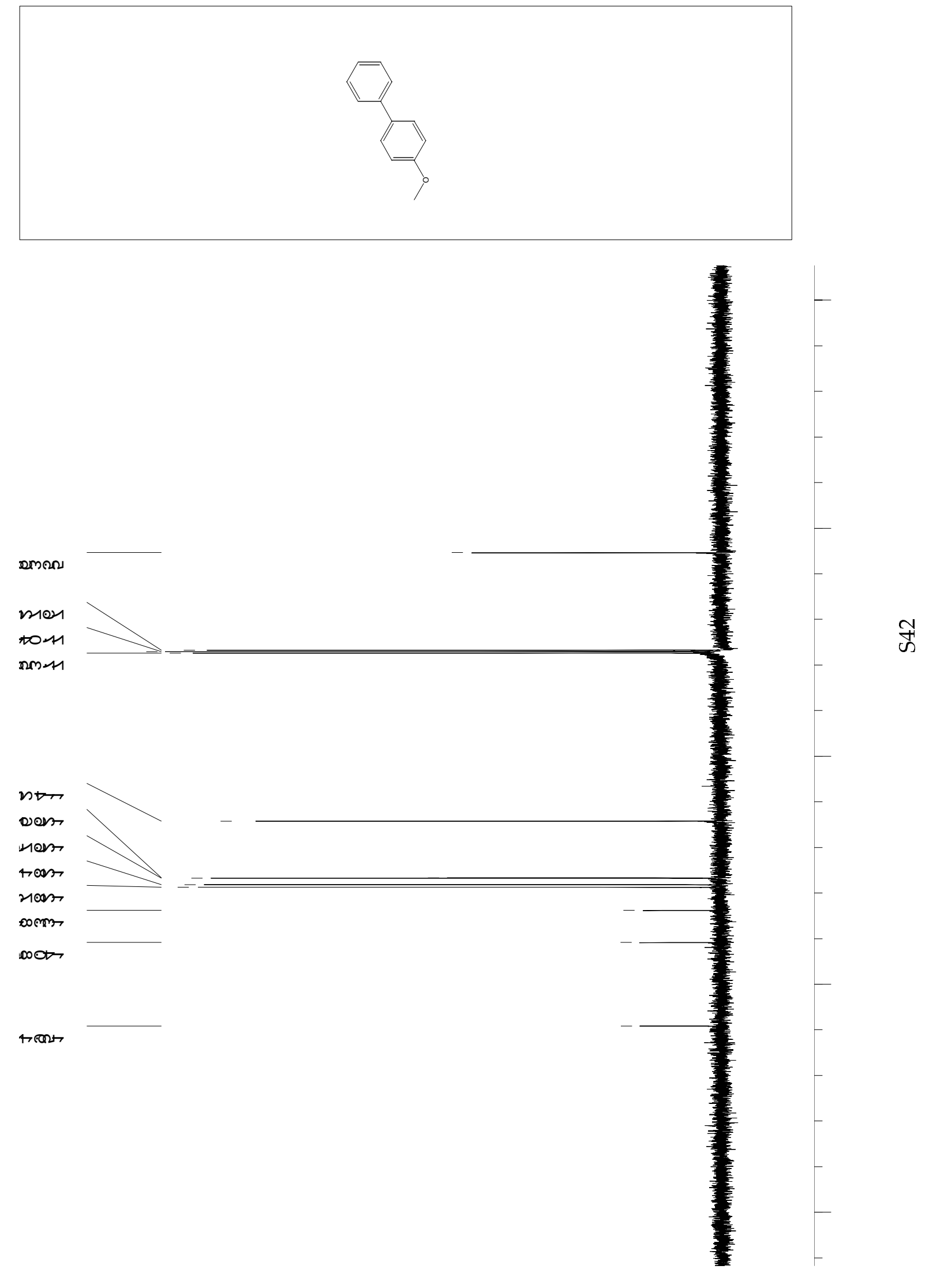

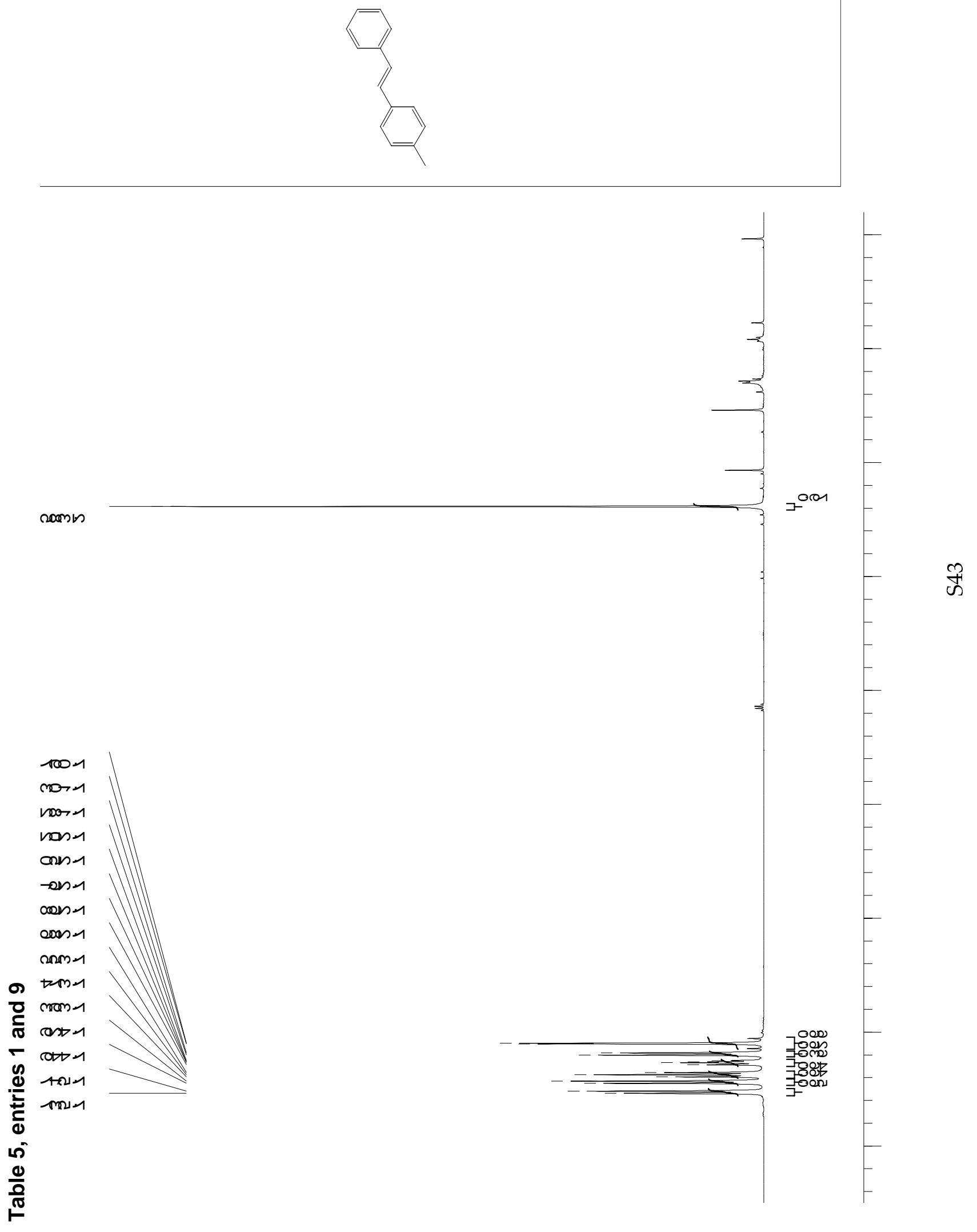


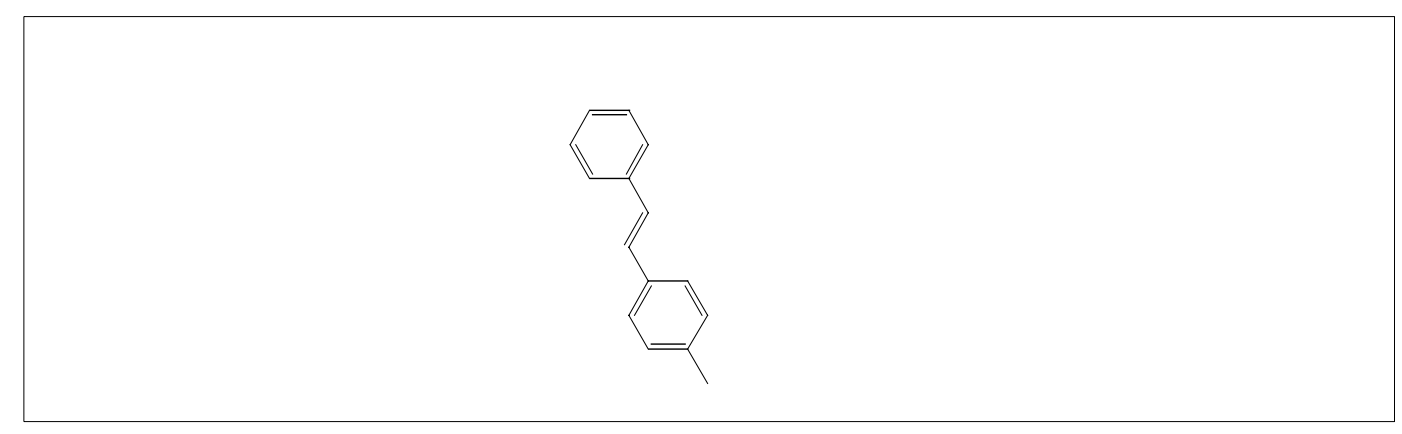

costs

then

not

IMA
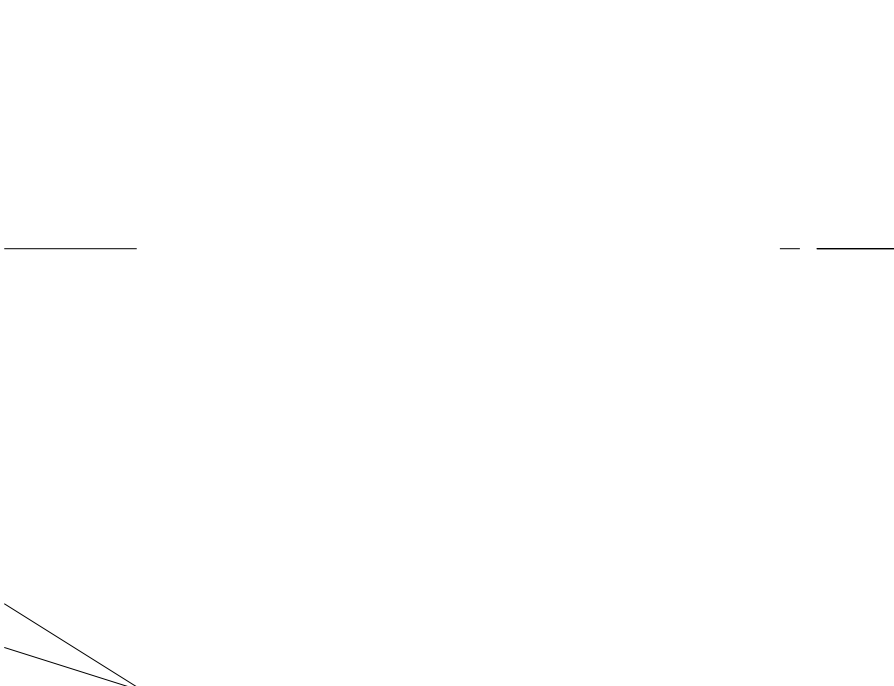

דas

tous

का

IAs

Does

stas

Nस

Dयक

Dयक 


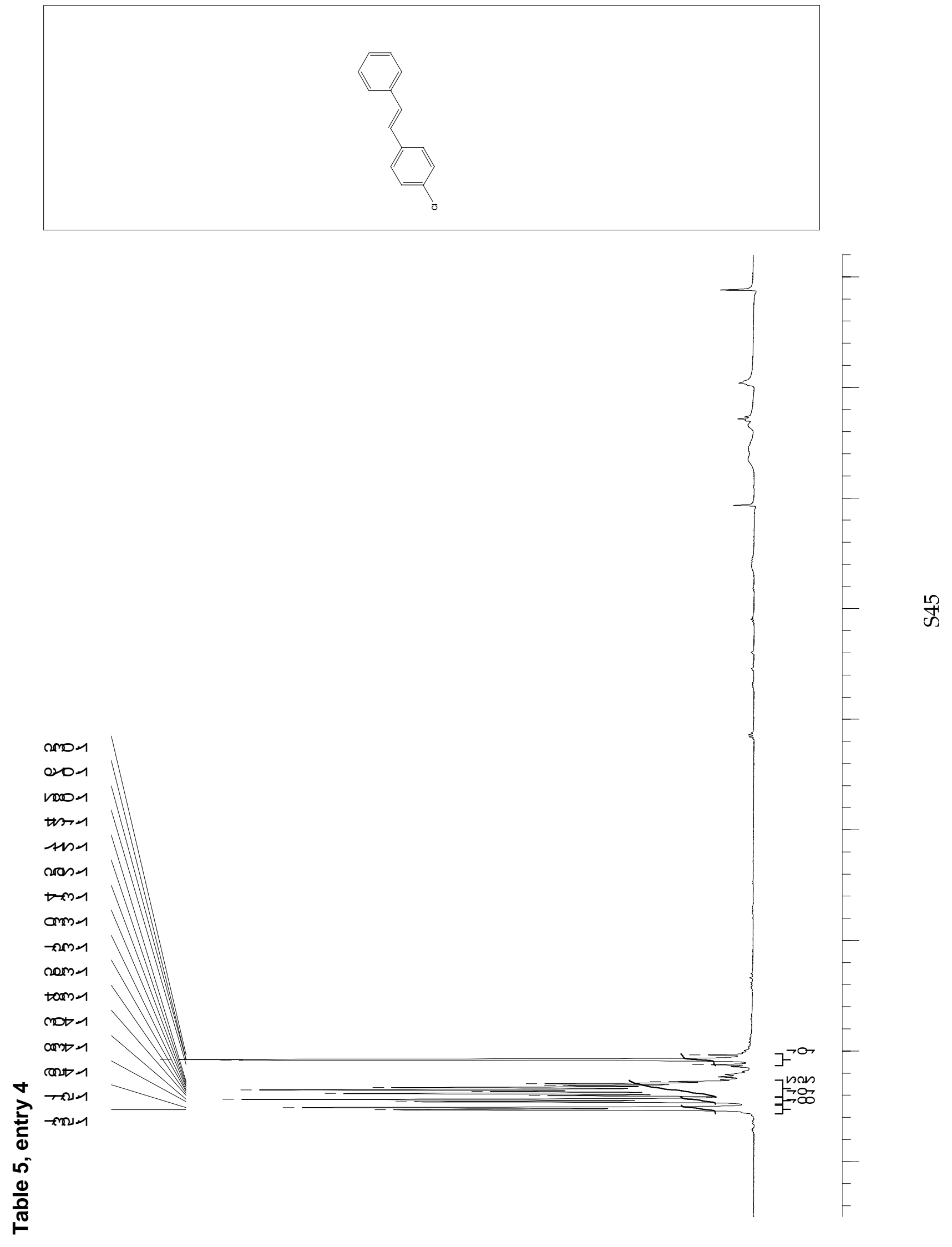



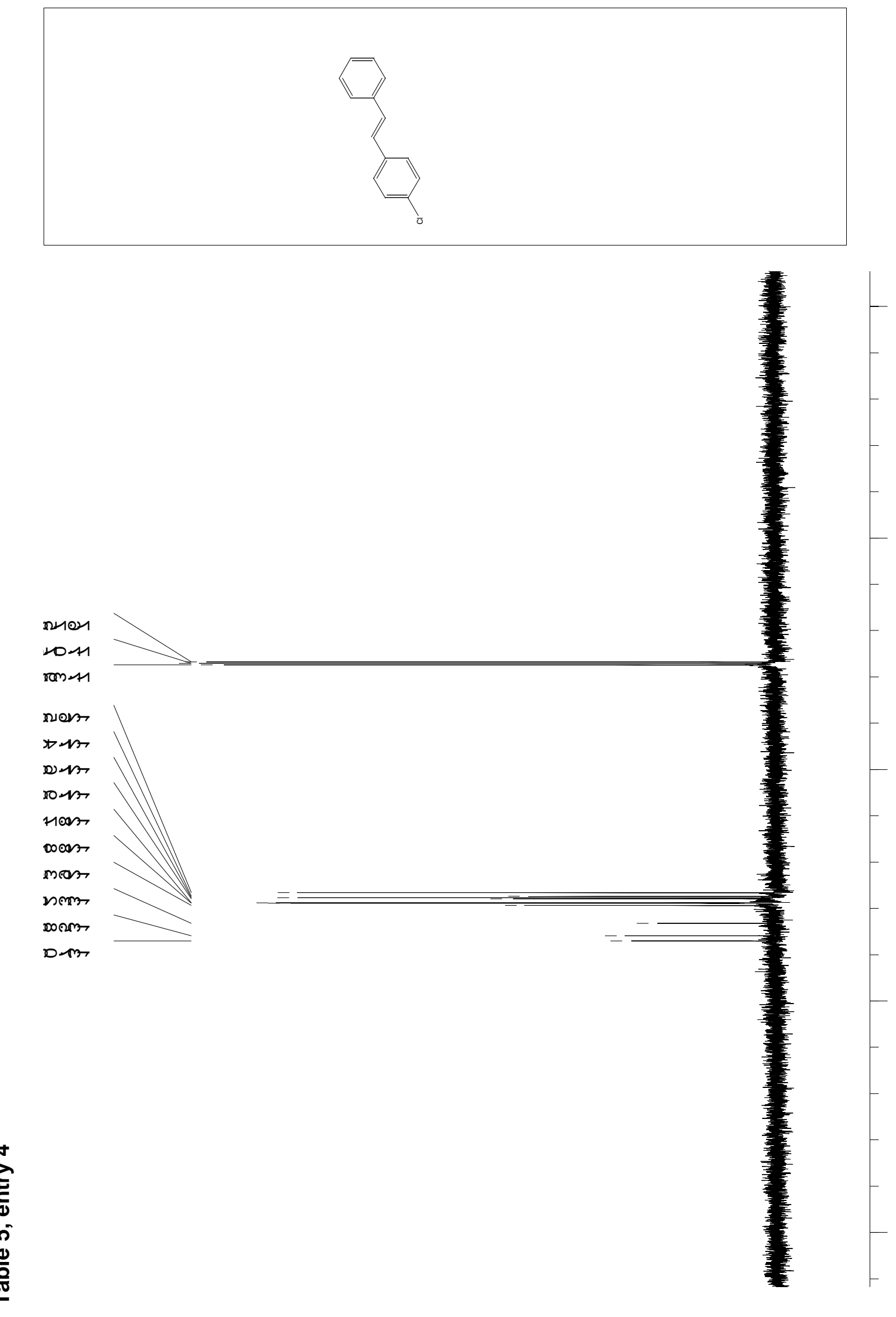

암 